\title{
Shear-driven kinetic Alfven wave in the plasma sheet boundary layer
}

\author{
B. V. Tiwari, R. Mishra, P. Varma, and M. S. Tiwari \\ Department of Physics and Electronics, Dr H S Gour University, Sagar, (M P) 470 003, India
}

(Received April 10, 2007; Revised November 2, 2007; Accepted December 17, 2007; Online published March 3, 2008)

\begin{abstract}
Shear-driven kinetic Alfven waves (KAWs) at the plasma sheet boundary layer (PSBL) were investigated for substorm events in the presence of a parallel electric field using the general loss-cone distribution function. Using a kinetic approach, we derived the expressions for dispersion relation and growth length of the KAW in the presence of the parallel electric field using the general loss-cone distribution function for both, weak and strong shear regimes. The frequency of the KAW obtained is in agreement with observed values of $0.1-4 \mathrm{~Hz}$ in the PSBL. The results explain the generation of the energetic KAWs at the PSBL by the shear at substorm onset. The parallel electric field associated with the energetic KAWs at substorm onset may heat the field-aligned electrons, leading to parallel electron energisation that ultimately causes an intense aurora. The electric field along magnetic field lines enhances the frequency of KAW but decreases the growth length in the case of weak shear. We also found that the parallel electric field can reflect KAW towards the PSBL. The loss-cone distribution index changes the profiles of frequency and growth length plots of the shear-driven KAW. Hence, the loss-cone distribution function is an important factor in the excitation of KAW in the active region of the magnetosphere, such as the PSBL and the auroral acceleration region.
\end{abstract}

Key words: Kinetic Alfven wave, velocity shear, loss-cone distribution function, parallel electric field, plasma sheet boundary layer, magnetic substorm.

\section{Introduction}

A magnetic substorm is a transient process that leads to a reconfiguration of the magnetotail. The occurrence of auroral arcs during the growth, expansion and recovery phases is a fundamental ionospheric signature of magnetospheric substorms (Keiling et al., 2002). A magnetospheric substorm involves the relocation of plasma and the flow of large amounts of energy in the magnetotail (Keiling et al., 2000). A large amount of this energy is eventually deposited in the ionosphere. The plasma sheet boundary layer (PSBL), which is located between the auroral acceleration region and the distant magnetotail, is of great importance in auroral substorm physics as it is regarded as a region of strong energy transfer between the distant tail and the auroral ionosphere (Eastman et al., 1984).

Keiling et al. (2002) have shown that very large amplitude electric fields $\left(E_{\perp}>100 \mathrm{mV} / \mathrm{m}\right)$ observed in the plasma sheet boundary layer (PSBL) by the Polar satellite are consistent with the propagation of Alfven waves along converging magnetic field lines. Wygant et al. (2000) have shown that the perpendicular electric fields in the PSBL at 4-6 $R_{E}\left(R_{E}\right.$ is Earth's radius) are associated with Alfven waves which carry large and sufficient Poynting flux towards the ionosphere to power magnetically conjugate auroral emissions.

Wygant et al. (2000, 2002) also reported evidence of small-scale, large-amplitude KAWs at the PSBL at alti-

Copyright (c) The Society of Geomagnetism and Earth, Planetary and Space Sciences (SGEPSS); The Seismological Society of Japan; The Volcanological Society of Japan; The Geodetic Society of Japan; The Japanese Society for Planetary Sciences; TERRAPUB. tudes of 4-6 $R_{E}$. The small-scale spikes, with a duration of $250 \mathrm{~ms}$ to $1 \mathrm{~s}$, have electric field amplitudes of up to 300 $\mathrm{mV} / \mathrm{m}$ and associated magnetic field variations between 0.5 to $5 \mathrm{nT}$. The large-scale KAWs have periods of 20-60 s. Similar wave activity has been seen from the Cluster satellite, and it has been verified with direct multipoint measurements that these waves have scales perpendicular to the magnetic field of the order of the electron inertial length (Lysak and Lotko, 1996).

Parallel electric fields are often observed in the upward current portion of the auroral acceleration region. Hull et al. (2003) reported 64 events in the upward current part of the auroral acceleration region, which contained microscopic parallel electric fields ranging in amplitude from about 25 to $300 \mathrm{mV} / \mathrm{m}$, as measured by the electric field instrument of Polar satellite.

Observations of large parallel electric fields from the FAST satellite indicate that such fields are found in regions of strong Alfvenic activity (Ergun et al., 2001). More recently, the Polar satellite has also observed dominant parallel electric field signatures with amplitudes of up to $150 \mathrm{mV} / \mathrm{m}$ that were associated with the large-amplitude Alfven waves in the PSBL during the sustorms (Wygant et al., 2000; Keiling et al., 2005).

Both observations and global magnetohydrodynamic (MHD) simulations have revealed that velocity shear can build up in the magnetosphere due to solar wind compression (Gavrishchaka et al., 1996). This is especially pertinent to the space plasma environment where increasing stress during geomagnetically active periods can translate into large velocity gradients. The PSBL is often charac- 
terised by a velocity shear, which intensifies during active periods (Gavrishchaka et al., 1996, and references therein). The free energy associated with this velocity gradient can excite waves in a broad range of frequencies and wavelengths (Gavrishchaka et al., 1996). Large flow shear in the PSBL has been also predicted in global MHD simulations because of the pressure gradient created as a consequence of the reconnection interacting with the ambient field. Nakamura et al. (2004) recently reported observations of transient strong flow shear events accompanied by energetic ion beams and localised field-aligned currents at the boundary of plasma sheet by Cluster and Geotail satellites. Inhomogeneties in plasma parameters, such as the density and drifts, which are inescapable in both space and laboratory plasma, naturally affect wave and plasma properties. A sharp density gradient in conjunction with shear flow configurations have been observed in and above the auroral region by the Freja satellite (Hamrin et al., 2001). This density gradient of the plasma particles has been observed to act as a source of free energy for many kinds of instabilities. All of these factors are important to the auroral processes, which may be the focus of future investigations.

Plasma particles in the PSBL that are imbedded with curved and converging field lines have very high anisotropies in their transverse and parallel velocity components (Wygant et al., 2002; Angelopoulos et al., 2002; Keiling et al., 2005) and, therefore, considerably depart from a Maxwellian distribution; instead, they have the losscone distribution of particles. Consequently, the use of isotropic Maxwellian distribution function is far from being a realistic equilibrium distribution function in this case. In the study reported here, the loss-cone distribution function (Summers and Thorne, 1995) is used to study the KAW driven by velocity shear. The loss-cone distribution function represents an anisotropy in the transverse and parallel velocity component. Wygant et al. (2002) and Angeloponlos et al. (2002) have shown that both ions and electrons have temperature anisotropies in the PSBL. Therefore, the use of the loss-cone distribution function for the ions and the electrons may be appropriate for this study. The importance of using the loss-cone distribution function for the plasma particles in the active regions of the magnetosphere, such as the auroral acceleration region and the PSBL, has been pointed out by various researchers (Gomberoff and Cuperman, 1981; Duan et al., 2005; Mishra and Tiwari, 2006; Ahirwar et al., 2006).

The purpose of our study was to study the shear-driven KAW in the presence of the parallel electric field using the loss-cone distribution function within the framework of simultaneous observations at the substorm-related KAWs in the PSBL, downward flowing electrons and upward flowing ions. The parallel electric field considered in our study is external in nature. The analysis is based on the kinetic model of the shear-driven kinetic Alfven wave developed by Wang et al. (1998). In Section 2, we derive expressions for the dispersion relation and growth length of the KAW for the weak and strong shear regimes in the presence of the parallel electric field and using the loss-cone distribution of plasma particles. In the Results and Discussion section, we graphically analyse the expressions derived for the disper- sion relation and growth length using recent satellite data and interpret the results to account for the recent observations of KAW in the PSBL. We close this article with our conclusions.

\section{Assumptions}

The basic assumptions are same as those made by Wang et al. (1998). The ambient magnetic field and flow velocity are along the $z$ direction and the velocity spatial variation is only in the $x$ direction i.e., $B=B_{0} \hat{e}_{z}, V_{0}=V_{0}(x) \hat{e}_{z}$. The external electric field $\left(E_{0}\right)$ is parallel to the magnetic field, i.e. along the $z$ direction. The perturbed quantities are assumed to vary as $\sim \exp -\left[i\left(k_{\perp} y+k_{z} z-\omega t\right)\right]$. The unperturbed distribution function is given by

$$
\begin{aligned}
F_{\alpha}^{(0)}= & \frac{n_{0}(X) v_{\perp}^{2 J}}{\pi v_{t \alpha \perp}^{2(J+1)}(X) J ! \sqrt{\pi} v_{t z \alpha c}} \\
& \times \exp \left[-\frac{v_{\perp}^{2}}{v_{t \alpha \perp}^{2}(X)}-\frac{\left[v_{z}-V_{0}(X)\right]^{2}}{v_{t \alpha z c}^{2}(X)}\right]
\end{aligned}
$$

where $n_{0}$ is the density of the plasma particles, and $\alpha=i, e$; $v_{\perp}^{2}=v_{x}^{2}+v_{y}^{2} . v_{z}$ and $X=x+\left(v_{y} / \omega_{c \alpha}\right)$ are the constants of motion.

$$
v_{t \alpha \perp}=\sqrt{\frac{2 T_{\alpha \perp}(J+1)^{-1}}{m_{\alpha}}}
$$

is the perpendicular thermal velocity.

$$
v_{t \alpha z c}=\left[\frac{2}{m_{\alpha}} T_{z \alpha c} R_{\alpha}\left(\cos \theta_{\alpha}+i \sin \theta_{\alpha}\right)\right]^{\frac{1}{2}}
$$

is the modified parallel thermal velocity

$$
\begin{aligned}
& \text { with } R_{\alpha}^{2}=1+\left(\frac{e E_{0}}{k_{z} T_{z \alpha c}}\right)^{2} \\
& \text { and } \theta_{\alpha}=\tan ^{-1}\left(\frac{e E_{0}}{k_{z} T_{z \alpha c}}\right) \\
& T_{z \alpha c}=T_{z \alpha}\left(1 \mp \frac{i e E_{0}}{k_{z} T_{\alpha z}}\right)
\end{aligned}
$$

is the modified ion and electron temperatures parallel to the magnetic field, which has been discussed in detail by Mishra and Tiwari (2006) and Ahirwar et al. (2006): $\omega_{c \alpha}$ is the cyclotron frequency, $\alpha$ is either ion or electron.

$J=0,1,2$ is the distribution index, also known as the steepness of the loss-cone. It characterises the width of the loss-cone. The width of the loss-cone decreases with increasing values of the index $J$. For $J=0$, this distribution represents a bi-Maxwellian distribution, and for $J=\infty$, this reduces to the Dirac-Delta function. Moreover, $f_{\perp}\left(v_{\perp}\right)$ peaks at about $J^{\frac{1}{2}} v_{t \perp}$ and has a half width of $\Delta v_{\perp} \sim$ $J^{\frac{-1}{2}} v_{t \perp}$. (Gomberoff and Cuperman, 1981; Summers and Thorne, 1995; Duan et al., 2005; Mishra and Tiwari, 2006; Ahirwar et al., 2006).

Expanding at $x=0$ gives (Wang et al., 1998)

$$
\begin{aligned}
& F_{\alpha}^{(0)}=f_{\alpha}^{(0)}\left\{1-\left[\frac{\left(v_{z}-V_{0}\right) V_{0} \kappa_{v}}{v_{t \alpha z c}^{2}}\right]\left(v_{y} / \omega_{c \alpha}\right)\right\} \\
& f_{\alpha}^{(0)}=\frac{n_{0} v_{\perp}^{2 J}}{\pi v_{t \alpha \perp}^{2(J+1)} J ! \sqrt{\pi} v_{t \alpha z}}
\end{aligned}
$$




$$
\begin{aligned}
& \times \exp \left[-\frac{v_{\perp}^{2}}{v_{t \alpha \perp}^{2}}-\frac{\left[v_{z}-V_{0}(x)\right]^{2}}{v_{t \alpha z}^{2}}\right] \\
& \text { where } \quad \kappa_{v}=\frac{1}{V_{0}(x)} \frac{d V_{0}(x)}{d x} \\
& \text { so that } \quad \frac{\partial F_{x}^{(0)}}{\partial x}=-\frac{\left[\left(v_{z}-V_{0}\right) V_{0} \kappa_{v}\right]}{v_{t \alpha z c}^{2}} f_{\alpha}^{(0)}
\end{aligned}
$$

The perturbations are considered in a plasma where only the low-frequency oscillations $\left(\omega \ll \omega_{c \alpha}\right)$ with the wave vector $\vec{k}$ approximately perpendicular to $B_{0}$ are important (Wang et al., 1998). The expression for $T_{z \alpha c}$ is originally derived by Pines and Schrieffer (1961) adopting the rigorous treatment of the kinetic approach for the collective behaviour of solid-state plasma. These researchers arrived at the result that the parallel electric field $\left(E_{0}\right)$ is eliminated by adopting the expression for $T_{z \alpha c}$ as expressed above (Pines and Schrieffer, 1961). The applied electric field parallel to $B_{0}$ modifies the electron thermal velocity in that direction and so that the parallel temperature $\left(T_{z e}\right)$ modifies to the complex temperature $T_{z e c}$. Here, a technique of Pines and Schrieffer (1961) is followed where a change in the distribution function is due to the change in the temperature parallel to the electric field. Mishra and Tiwari (2006) and Ahirwar et al. (2006) have considered this method in detail for the investigation of electrostatic and electromagnetic ion cyclotron instability, respectively.

\section{Dispersion Relation}

Using the mathematical analysis of Wang et al. (1998), the dispersion relation for the shear-driven KAW in the presence of the parallel electric field and loss-cone distribution function is derived as

$$
\begin{aligned}
& \left(\varepsilon_{c}^{2} \varpi^{2}-k_{z}^{2} v_{A}^{2} \frac{(J+1) \lambda_{i}}{1-\Pi_{0}}+\frac{k_{z} k_{\perp} V_{0}}{\omega_{c i}} \frac{(J+1) v_{t i \perp}^{2}}{2}\right) \\
& \times\left[\left(1+\frac{k_{\perp} V_{0} \kappa_{v}}{k_{z} \omega_{c e}}\right)\left(1+i \sqrt{\frac{\pi}{2}} \frac{\varpi}{k_{z} v_{t e z c}}\right)\right. \\
& \left.\quad-\Pi_{0}\left(1-\frac{k_{\perp} V_{0} \kappa_{v}}{k_{z} \omega_{c i}}\right) \frac{k_{z}^{2} v_{t i z c}^{2}}{\varpi^{2}} \frac{T_{e}}{T_{i}}\right] \\
& -\frac{T_{e}}{T_{i}}(J+1) \lambda_{i} k_{z}^{2} v_{A}^{2}=0
\end{aligned}
$$

is the modified ion thermal anisotropy

where $\varepsilon=\frac{v_{t i \perp}^{2}}{v_{t i z}^{2}}$ (Gomberoff and Cuperman, 1981;

Summers and Thorne, 1995)

$v_{t \alpha z c}^{2}$ and $T_{z \alpha c}$ are given by Eqs. (3) and (6), respectively.

$\varpi$ is the Doppler shifted frequency of the wave, $v_{A}$ is the Alfven velocity, $k_{\perp}$ and $k_{z}$ are the perpendicular and parallel wave vectors, respectively, $m_{i}$ and $m_{e}$ are the ion and electron mass, respectively, $T_{i}$ and $T_{e}$ are the ion and electron temperature, respectively,

$$
\begin{aligned}
& \text { and } \quad \lambda_{i}=\frac{k_{\perp}^{2} v_{t i \perp}^{2}}{\omega_{c i}^{2}} \\
& \Pi_{0}=\exp \left(-\lambda_{i}\right) I_{0}\left(\lambda_{i}\right) \approx 1-(J+1) \lambda_{i}
\end{aligned}
$$

with $I_{0}\left(\lambda_{i}\right)$ as the modified Bessel's function.

The small imaginary term of the order of $\frac{\omega}{k_{z} v_{t e z}}$ in the dispersion relation is due to the resonant electron, and the small term of the order of $\frac{k_{z}^{2} v_{t i z}^{2}}{\omega^{2}}$ is due to the ion longitudinal motion. Equation (10) shows that the parallel electric field $\left(E_{0}\right)$ modifies the dispersion relation of the shear-driven KAW.

For $E_{0}=0$ and $J=0$, Eq. (10) reduces to that given by Wang et al. (1998) and Tiwari et al. (2006).

Equation (10) may further be simplified as

$$
\begin{aligned}
& {\left[\omega^{2}(J+1)^{2}\left(\frac{v_{t i \perp}^{2}}{v_{t i z}^{2}}\right)^{2}\left(\cos 2 \theta_{i}+i \sin 2 \theta_{i}\right)\right.} \\
& \left.-k_{z}^{2} v_{A}^{2}\left(1-\frac{\alpha_{i} \beta_{i \perp}(J+1)}{2}\right)\right]\left[\left(1+\frac{m_{e}}{m_{i}} \alpha_{i}\right)\right. \\
& \times\left(1+i \sqrt{\frac{\pi m_{e}}{4 T_{e z} R_{e}}} v_{p z}\left(\cos \frac{\theta_{e}}{2}-i \frac{\sin \theta_{e}}{2}\right)\right) \\
& \left.-\frac{\Pi_{0}\left(1-\alpha_{i}\right)}{v_{p z}^{2}}\left(\frac{T_{e}}{T_{i}}\right) \frac{2 T_{i z} R_{i}}{m_{i}}\left(\cos \theta_{i}-i \sin \theta_{i}\right)\right] \\
& -\frac{T_{e}}{T_{i}}(J+1) \lambda_{i} k_{z}^{2} v_{A}^{2}=0 \\
& \text { where } \quad \alpha_{i}=\frac{k_{\perp} V_{0} \beta_{v}}{k_{z} \omega_{c i} \rho_{c i}} \\
& \text { with } \beta_{v}=\kappa_{v} \rho_{c i}
\end{aligned}
$$

in which $\kappa_{v}$ is given by Eq. (8b) and $\rho_{c i}$ is the ion gyroradius.

$$
\beta_{i \perp}=\frac{v_{t i \perp}^{2}}{v_{A}^{2}}
$$

$v_{p z}=\frac{\omega}{k_{z}}$ is the phase velocity.

Separating its real and imaginary parts, we obtain

$$
\begin{aligned}
& I_{m} D(\omega, k)=\left(1+\frac{m_{e}}{m_{i}} \alpha_{i}\right) \frac{(J+1)^{2}\left(\frac{v_{t i \perp}^{2}}{v_{t i z}^{2}}\right)^{2} \omega^{5}}{k_{z} R_{i}^{2}} \\
& \times \sqrt{\frac{\pi m_{e}}{4 T_{e z} R_{e}}} \cos \left(2 \theta_{i}-\frac{\theta_{e}}{2}\right)-\left(1+\frac{m_{e}}{m_{i}} \alpha_{i}\right) \\
& \times \frac{(J+1)^{2}\left(\frac{v_{t i \perp}^{2}}{v_{t i z}^{2}}\right)^{2} \omega^{4}}{R_{i}^{2}} \sin 2 \theta_{i} \\
& -\left(1+\frac{m_{e}}{m_{i}} \alpha_{i}\right) \frac{\omega^{3}}{k_{z}} \sqrt{\frac{\pi m_{e}}{4 T_{e z} R_{e}}} k_{z}^{2} v_{A}^{2}\left(1-\frac{\alpha_{i} \beta_{i \perp}(J+1)}{2}\right) \\
& \times \cos \frac{\theta_{e}}{2}-\omega^{2} \Pi_{0}\left(1-\alpha_{i}\right) \frac{2(J+1)^{2}\left(\frac{v_{t i \perp}^{2}}{v_{t i z}^{2}}\right)^{2} k_{z}^{2}}{R_{i}}\left(\frac{T_{e}}{T_{i}}\right) \\
& \times \frac{T_{i z}}{m_{i}} \sin \theta_{i}-\Pi_{0}\left(1-\alpha_{i}\right) k_{z}^{2}\left(\frac{T_{e}}{T_{i}}\right) \\
& \times \frac{2 T_{i z} R_{i}}{m_{i}} k_{z}^{2} v_{A}^{2}\left(1-\frac{\alpha_{i} \beta_{i \perp}(J+1)}{2}\right) \sin \theta_{i} \\
& R_{e} D(\omega, k)=-\left[\left(1+\frac{m_{e}}{m_{i}} \alpha_{i}\right) \frac{(J+1)^{2}\left(\frac{v_{t i \perp}^{2}}{v_{t i z}^{2}}\right)^{2}}{k_{z} R_{i}^{2}}\right. \\
& \left.\times \sqrt{\frac{\pi m_{e}}{4 T_{e z} R_{e}}} \sin \left(2 \theta_{i}-\frac{\theta_{e}}{2}\right)\right] \omega^{5}
\end{aligned}
$$




$$
\begin{aligned}
& +\left[\left(1+\frac{m_{e}}{m_{i}} \alpha_{i}\right) \frac{(J+1)^{2}\left(\frac{v_{t i \perp}^{2}}{v_{t i z}^{2}}\right)^{2}}{R_{i}^{2}} \cos 2 \theta_{i}\right] \omega^{4} \\
& -\left[\left(1+\frac{m_{e}}{m_{i}} \alpha_{i}\right) \frac{k_{z}^{2} v_{A}^{2}}{k_{z}}\left(1-\frac{\alpha_{i} \beta_{i \perp}(J+1)}{2}\right)\right. \\
& \left.\times \sqrt{\frac{\pi m_{e}}{4 T_{e z} R_{e}}} \sin \frac{\theta_{e}}{2}\right] \omega^{3} \\
& -\left[\left(1+\frac{m_{e}}{m_{i}} \alpha_{i}\right) k_{z}^{2} v_{A}^{2}\left(1-\frac{\alpha_{i} \beta_{i \perp}(J+1)}{2}\right)\right] \omega^{2} \\
& -\left[\Pi_{0}\left(1-\alpha_{i}\right) \frac{k_{z}^{2}(J+1)^{2}\left(\frac{v_{t i \perp}^{2}}{v_{t i z}^{2}}\right)^{2}\left(\frac{T_{e}}{T_{i}}\right) \frac{2 T_{i z}}{m_{i}} \cos \theta_{i}}{R_{i}}\right. \\
& \left.-\left(\frac{T_{e}}{T_{i}}\right)(J+1) \lambda_{i} k_{z}^{2} v_{A}^{2}\right] \omega^{2} \\
& +\Pi_{0}\left(1-\alpha_{i}\right) k_{z}^{4} v_{A}^{2}\left(1-\frac{\alpha_{i} \beta_{i \perp}(J+1)}{2}\right) \frac{T_{e}}{T_{i}} \frac{2 T_{i z} R_{i}}{m_{i}} \cos \theta_{i}
\end{aligned}
$$

\subsection{Case of weak shear}

In the weak shear regime-i.e., during the onset and release of the magnetic substorm- $\rho_{i} \gg L$ where $\rho_{i}$ is the ion gyro-radius and $L$ is the velocity shear scale length and $\frac{d V_{0}}{d x} \ll \Omega_{H}$ where $V_{0}$ is the ion flow velocity parallel to the magnetic field, $\frac{d V_{0}}{d x}$ is the transverse velocity gradient, which also represents the velocity shear, and $\Omega_{H}$ is the ion cyclotron frequency (Gavrishchaka et al., 2000; Ganguli et al., 2002; Scime et al., 2003).

For the case of weak shear, the instability boundary is (Wang et al., 1998)

$$
1<\alpha_{i}<2 / \beta_{i \perp}
$$

where $\alpha_{i}$ and $\beta_{i \perp}$ are given by Eqs. (16) and (17), respectively.

Considering the imaginary part of Eq. (10)-i.e. Eq. (18) - and using Eqs. (2), (12), (13) and (14), we derive the dispersion relation for the weak shear-driven KAW in the presence of the parallel electric field as

$$
\begin{aligned}
& \left(1+\frac{m_{e}}{m_{i}} \alpha_{i}\right) \frac{(J+1)^{2}\left(\frac{v_{t i \perp}^{2}}{v_{t i z}^{2}}\right)^{2} \omega^{5}}{k_{z} R_{i}^{2}} \\
& \times \sqrt{\frac{\pi m_{e}}{4 T_{e z} R_{e}}} \cos \left(2 \theta_{i}-\frac{\theta_{e}}{2}\right) \\
& -\left(1+\frac{m_{e}}{m_{i}} \alpha_{i}\right) \frac{(J+1)^{2}\left(\frac{v_{t i \perp}^{2}}{v_{t i z}^{2}}\right)^{2} \omega^{4}}{R_{i}^{2}} \sin 2 \theta_{i} \\
& -\left(1+\frac{m_{e}}{m_{i}} \alpha_{i}\right) \frac{\omega^{3}}{k_{z}} \sqrt{\frac{\pi m_{e}}{4 T_{e z} R_{e}}} k_{z}^{2} v_{A}^{2} \\
& \times\left(1-\frac{\alpha_{i} \beta_{i \perp}(J+1)}{2}\right) \cos \frac{\theta_{e}}{2} \\
& -\omega^{2} \Pi_{0}\left(1-\alpha_{i}\right) \frac{2(J+1)^{2}\left(\frac{v_{t i \perp}^{2}}{v_{t i z}^{2}}\right)^{2} k_{z}^{2}}{R_{i}}\left(\frac{T_{e}}{T_{i}}\right) \frac{T_{i z}}{m_{i}} \sin \theta_{i} \\
& -\Pi_{0}\left(1-\alpha_{i}\right) k_{z}^{2}\left(\frac{T_{e}}{T_{i}}\right) \frac{2 T_{i z} R_{i}}{m_{i}} k_{z}^{2} v_{A}^{2}
\end{aligned}
$$

$$
\times\left(1-\frac{\alpha_{i} \beta_{i \perp}(J+1)}{2}\right) \sin \theta_{i}=0
$$

Equation (21) shows that in the weak shear regime the parallel electric field and the loss-cone distribution index modify the dispersion relation of the shear-driven KAW. The loss-cone distribution function affects the dispersion relation through the perpendicular thermal velocity, while the parallel electric field affects the dispersion relation through the complex parallel thermal velocity which is expressed in terms of $R \alpha$ and $\theta \alpha$ given by Eqs. (4) and (5), respectively. The shear affects the dispersion relation through the factor $\alpha_{i}$.

For $E_{0}=0$ and $J=0$, Eq. (21) reduces to that given by Wang et al. (1998) and Tiwari et al. (2006).

\subsection{Case of strong shear}

In the strong shear regime-i.e. during the expansion phase of the magnetic substorm- $\rho_{i} \ll L$ and $\frac{d V_{0}}{d x} \gg \Omega_{H}$ (Gavrishchaka et al., 2000; Ganguli et al., 2002; Scime et al., 2003).

For the case of strong shear, the instability boundary is (Wang et al., 1998)

$$
\alpha_{i}>2 / \beta_{i \perp}
$$

where $\alpha_{i}$ and $\beta_{i \perp}$ are given by Eqs. (16) and (17), respectively.

Again, considering the imaginary part of Eq. (10)-i.e. Eq. (18) — and applying condition (22), the dispersion relation for the strong shear-driven KAW in the presence of the parallel electric field is derived as

$$
\begin{aligned}
& \left(1+\alpha_{e}\right) \frac{(J+1)^{2}\left(\frac{v_{t i \perp}^{2}}{v_{t i z}^{2}}\right)^{2} \omega^{5}}{k_{z} R_{i}^{2}} \sqrt{\frac{\pi m_{e}}{4 T_{e z} R_{e}}} \cos \left(2 \theta_{i}-\frac{\theta_{e}}{2}\right) \\
& -\left(1+\alpha_{e}\right) \frac{(J+1)^{2}\left(\frac{v_{t i \perp}^{2}}{v_{t i z}^{2}}\right)^{2} \omega^{4}}{R_{i}^{2}} \sin 2 \theta_{i} \\
& -\left(1+\alpha_{e}\right) \frac{\omega^{3}}{k_{z}} \sqrt{\frac{\pi m_{e}}{4 T_{e z} R_{e}}} k_{z}^{2} v_{A}^{2}\left(1+\frac{m_{i}}{m_{e}} \frac{\alpha_{e} \beta_{i \perp}(J+1)}{2}\right) \\
& \times \cos \frac{\theta_{e}}{2}-\omega^{2} \Pi_{0}\left(1+\frac{m_{i}}{m_{e}} \alpha_{e}\right) \\
& \times \frac{2(J+1)^{2}\left(\frac{v_{t i \perp}^{2}}{v_{t i z}^{2}}\right)^{2} k_{z}^{2}}{R_{i}}\left(\frac{T_{e}}{T_{i}}\right) \frac{T_{i z}}{m_{i}} \sin \theta_{i} \\
& -\Pi_{0}\left(1+\frac{m_{i}}{m_{e}} \alpha_{e}\right) k_{z}^{2}\left(\frac{T_{e}}{T_{i}}\right) \frac{2 T_{i z} R_{i}}{m_{i}} k_{z}^{2} v_{A}^{2} \\
& \times\left(1+\frac{m_{i}}{m_{e}} \frac{\alpha_{e} \beta_{i \perp}(J+1)}{2}\right) \sin \theta_{i}=0
\end{aligned}
$$

where $\alpha_{e}=\frac{k_{\perp} d V_{0} / d x}{k_{z} \omega_{c e}}$.

Equation (23) shows that the parallel electric field and the loss-cone distribution index $(J)$ modify the dispersion relation of the shear-driven KAW in the strong shear regime as well. The loss-cone distribution function affects the dispersion relation through the perpendicular thermal velocity, while the parallel electric filed affects the dispersion relation through the complex parallel thermal velocity which is expressed in terms of $R \alpha$ and $\theta \alpha$ given by Eqs. (4) and 
(5) respectively. The shear affects the dispersion relation through the factor $\alpha_{e}$.

For $E_{0}=0$ and $J=0$, Eq. (23) reduces to that given by Wang et al. (1998) and Tiwari et al. (2006).

\section{Growth Rate of the Wave}

\subsection{Case of weak shear}

The growth rate of the KAW in the weak shear regime is derived from

$$
\gamma=\frac{R_{e} D(\omega, k)}{\frac{\partial}{\partial \omega} I_{m} D(\omega, k)}
$$

Using Eqs. (18) and (19), we derive the growth rate as

$$
\begin{aligned}
& \gamma=\left\{-\left[\left(1+\frac{m_{e}}{m_{i}} \alpha_{i}\right) \frac{(J+1)^{2}\left(\frac{v_{t i \perp}^{2}}{v_{t i z}^{2}}\right)^{2}}{k_{z} R_{i}^{2}}\right.\right. \\
& \left.\times \sqrt{\frac{\pi m_{e}}{4 T_{e z} R_{e}}} \sin \left(2 \theta_{i}-\frac{\theta_{e}}{2}\right)\right] \omega^{5} \\
& +\left[\left(1+\frac{m_{e}}{m_{i}} \alpha_{i}\right) \frac{(J+1)^{2}\left(\frac{v_{t i \perp}^{2}}{v_{t i z}^{2}}\right)^{2}}{R_{i}^{2}} \cos 2 \theta_{i}\right] \omega^{4} \\
& -\left[\left(1+\frac{m_{e}}{m_{i}} \alpha_{i}\right) \frac{k_{z}^{2} v_{A}^{2}}{k_{z}}\left(1-\frac{\alpha_{i} \beta_{i \perp}(J+1)}{2}\right)\right. \\
& \left.\times \sqrt{\frac{\pi m_{e}}{4 T_{e z} R_{e}}} \sin \frac{\theta_{e}}{2}\right] \omega^{3} \\
& -\left[\left(1+\frac{m_{e}}{m_{i}} \alpha_{i}\right) k_{z}^{2} v_{A}^{2}\left(1-\frac{\alpha_{i} \beta_{i \perp}(J+1)}{2}\right)\right] \omega^{2} \\
& -\left[\Pi_{0}\left(1-\alpha_{i}\right) \frac{k_{z}^{2}(J+1)^{2}\left(\frac{v_{t i \perp}^{2}}{v_{t i z}^{2}}\right)^{2}}{R_{i}}\left(\frac{T_{e}}{T_{i}}\right) \frac{2 T_{i z}}{m_{i}} \cos \theta_{i}\right. \\
& \left.-\left(\frac{T_{e}}{T_{i}}\right)(J+1) \lambda_{i} k_{z}^{2} v_{A}^{2}\right] \omega^{2} \\
& +\Pi_{0}\left(1-\alpha_{i}\right) k_{z}^{4} v_{A}^{2}\left(1-\frac{\alpha_{i} \beta_{i \perp}(J+1)}{2}\right) \\
& \left.\times \frac{T_{e}}{T_{i}} \frac{2 T_{i z} R_{i}}{m_{i}} \cos \theta_{i}\right\} \\
& \times\left[\left(1+\frac{m_{e}}{m_{i}} \alpha_{i}\right) \frac{5(J+1)^{2}\left(\frac{v_{t i \perp}^{2}}{v_{t i z}^{2}}\right)^{2} \omega^{4}}{k_{z} R_{i}^{2}}\right. \\
& \times \sqrt{\frac{\pi m_{e}}{4 T_{e z} R_{e}}} \cos \left(2 \theta_{i}-\frac{\theta_{e}}{2}\right) \\
& -\left(1+\frac{m_{e}}{m_{i}} \alpha_{i}\right) \frac{4(J+1)^{2}\left(\frac{v_{t i \perp}^{2}}{v_{t i z}^{2}}\right)^{2} \omega^{3}}{R_{i}^{2}} \sin 2 \theta_{i} \\
& -\left(1+\frac{m_{e}}{m_{i}} \alpha_{i}\right) \frac{3 \omega^{2}}{k_{z}} \sqrt{\frac{\pi m_{e}}{4 T_{e z} R_{e}}} k_{z}^{2} v_{A}^{2}
\end{aligned}
$$

$$
\begin{aligned}
& \times\left(1-\frac{\alpha_{i} \beta_{i \perp}(J+1)}{2}\right) \cos \frac{\theta_{e}}{2}-2 \omega \Pi_{0}\left(1-\alpha_{i}\right) \\
& \left.\times \frac{2(J+1)^{2}\left(\frac{v_{t \perp \perp}^{2}}{v_{t i z}^{2}}\right)^{2} k_{z}^{2}}{R_{i}}\left(\frac{T_{e}}{T_{i}}\right) \frac{T_{i z}}{m_{i}} \sin \theta_{i}\right]^{-1}
\end{aligned}
$$

Where $v_{t \alpha z c}, T_{z \alpha c}, \lambda_{i}, \alpha_{i}$ and $\beta_{i \perp}$ are given by Eqs. (3), (6), (13), (16) and (17), respectively.

Equation (25) shows that the external parallel electric field and the loss-cone distribution function modify the growth rate of the KAW in the weak shear regime. The parallel electric field affects the growth rate through the complex parallel thermal velocity, whereas the loss-cone affects it through the perpendicular thermal velocity. For $E_{0}=0$ and $J=0$, Eq. (25) reduces to that given by Wang et al. (1998) and Tiwari et al. (2006).

\subsection{Case of strong shear}

The growth rate for the KAW driven by strong shear is derived from

$$
\gamma=\frac{-I_{m} D(\omega, k)}{\frac{\partial}{\partial \omega} R_{e} D(\omega, k)}
$$

Again using Eqs. (18) and (19), we derive the growth rate as

$$
\begin{aligned}
& \gamma=\left[-\left(1+\alpha_{e}\right) \frac{(J+1)^{2}\left(\frac{v_{t i \perp}^{2}}{v_{t i z}^{2}}\right)^{2} \omega^{5}}{k_{z} R_{i}^{2}} \sqrt{\frac{\pi m_{e}}{4 T_{e z} R_{e}}}\right. \\
& \times \cos \left(2 \theta_{i}-\frac{\theta_{e}}{2}\right)+\left(1+\alpha_{e}\right) \frac{(J+1)^{2}\left(\frac{v_{t i \perp}^{2}}{v_{t i z}^{2}}\right)^{2} \omega^{4}}{R_{i}^{2}} \sin 2 \theta \\
& +\left(1+\alpha_{e}\right) \frac{\omega^{3}}{k_{z}} \sqrt{\frac{\pi m_{e}}{4 T_{e z} R_{e}}} k_{z}^{2} v_{A}^{2}\left(1-\frac{\alpha_{i} \beta_{i \perp}(J+1)}{2}\right) \cos \frac{\theta_{e}}{2} \\
& +\omega^{2} \Pi_{0}\left(1-\alpha_{i}\right) \frac{2(J+1)^{2}\left(\frac{v_{t i \perp}^{2}}{v_{t i z}^{2}}\right)^{2} k_{z}^{2}}{R_{i}}\left(\frac{T_{e}}{T_{i}}\right) \frac{T_{i z}}{m_{i}} \sin \theta_{i} \\
& +\Pi_{0}\left(1-\alpha_{i}\right) k_{z}^{2}\left(\frac{T_{e}}{T_{i}}\right) \frac{2 T_{i z} R_{i}}{m_{i}} k_{z}^{2} v_{A}^{2} \\
& \left.\times\left(1-\frac{\alpha_{i} \beta_{i \perp}(J+1)}{2}\right) \sin \theta_{i}\right] \\
& \times\left\{-5\left[\left(1+\frac{m_{e}}{m_{i}} \alpha_{i}\right) \frac{(J+1)^{2}\left(\frac{v_{t i \perp}^{2}}{v_{t i z}^{2}}\right)^{2}}{k_{z} R_{i}^{2}}\right.\right. \\
& \left.\times \sqrt{\frac{\pi m_{e}}{4 T_{e z} R_{e}}} \sin \left(2 \theta_{i}-\frac{\theta_{e}}{2}\right)\right] \omega^{4} \\
& +4\left[\left(1+\frac{m_{e}}{m_{i}} \alpha_{i}\right) \frac{(J+1)^{2}\left(\frac{v_{t i \perp}^{2}}{v_{t i z}^{2}}\right)^{2}}{R_{i}^{2}} \cos 2 \theta_{i}\right] \omega^{3} \\
& -3\left[\left(1+\frac{m_{e}}{m_{i}} \alpha_{i}\right) \frac{k_{z}^{2} v_{A}^{2}}{k_{z}}\left(1-\frac{\alpha_{i} \beta_{i \perp}(J+1)}{2}\right)\right. \\
& \left.\times \sqrt{\frac{\pi m_{e}}{4 T_{e z} R_{e}}} \sin \frac{\theta_{e}}{2}\right] \omega^{2}
\end{aligned}
$$




$$
\begin{aligned}
& -2\left[\left(1+\frac{m_{e}}{m_{i}} \alpha_{i}\right) k_{z}^{2} v_{A}^{2}\left(1-\frac{\alpha_{i} \beta_{i \perp}}{2(J+1)}\right)\right] \omega \\
& -2\left[\Pi_{0}\left(1-\alpha_{i}\right) \frac{k_{z}^{2}(J+1)^{2}\left(\frac{v_{t i \perp}^{2}}{v_{t i z}^{2}}\right)^{2}}{R_{i}}\left(\frac{T_{e}}{T_{i}}\right) \frac{2 T_{i z}}{m_{i}} \cos \theta_{i}\right. \\
& \left.\left.-\left(\frac{T_{e}}{T_{i}}\right)(J+1) \lambda_{i} k_{z}^{2} v_{A}^{2}\right] \omega\right\}^{-1}
\end{aligned}
$$

Here, $v_{t \alpha z c}, T_{z \alpha c}, \Pi_{0}, \alpha_{i}$ and $\beta_{i \perp}$ are given by Eqs. (3), (6), (14), (16) and (17), respectively.

The expression for the growth rate shows that the resonant electrons associated with the term $\left(i \sqrt{\frac{\pi}{2}} \frac{\omega}{k_{z} v_{t e z}}\right)$ act as a source of free energy for the KAW. For strong shear, the ion longitudinal motion associated with the term $v_{t i z}^{2}$ suppresses the instability. The external parallel electric field and the loss-cone distribution index modify the growth rate of the KAW. The parallel electric field affects the growth rate through the complex parallel thermal velocity, whereas the loss-cone affects it through the perpendicular thermal velocity. Temperature anisotropy and the density of the plasma particles also affect the growth rate of the wave.

For $E_{0}=0$ and $J=0$, Eq. (27) reduces to that given by Wang et al. (1998) and Tiwari et al. (2006).

\section{Growth Length of the Wave}

\subsection{Case of weak shear}

The growth length of the KAW in the weak shear regime is derived from

$$
L_{g}=\frac{v_{g}}{\gamma}
$$

Using Eq. (21), we derive the Group velocity $v_{g}$ as

$$
\begin{aligned}
& v_{g}=\left\{-\left[\left(\frac{m_{e}}{m_{i}} \frac{\alpha_{i}}{k_{\perp}}\right) \frac{(J+1)^{2}\left(\frac{v_{t i \perp}^{2}}{v_{t i z}^{2}}\right)^{2}}{k_{z} R_{i}^{2}}\right.\right. \\
& \left.\times \sqrt{\frac{\pi m_{e}}{4 T_{e z} R_{e}}} \cos \left(2 \theta_{i}-\frac{\theta_{e}}{2}\right)\right] \omega^{5} \\
& +\left[\left(\frac{m_{e}}{m_{i}} \frac{\alpha_{i}}{k_{\perp}}\right) \frac{(J+1)^{2}\left(\frac{v_{t i \perp}^{2}}{v_{t i z}^{2}}\right)^{2}}{R_{i}^{2}} \sin 2 \theta_{i}\right] \omega^{4} \\
& +\left[\frac{\alpha_{i}}{k_{\perp}} \frac{k_{z}^{2} v_{A}^{2}}{k_{z}}\left(\frac{m_{e}}{m_{i}}-\frac{\beta_{i \perp}(J+1)}{2}\left(1+\frac{2 m_{e}}{m_{i}}\right)\right)\right. \\
& \left.\left.\times \sqrt{\frac{\pi m_{e}}{4 T_{e z} R_{e}}} \cos \frac{\theta_{e}}{2}\right] \omega^{3}\right)^{3} \\
& +\left[\Pi_{0} \frac{\alpha_{i}}{k_{\perp}} \frac{k_{z}^{2}}{(J+1)^{2}\left(\frac{v_{t i \perp}^{2}}{v_{t i z}^{2}}\right)^{2}}\left(\frac{T_{e}}{T_{i}}\right) \frac{2 T_{i z}}{R_{i}} \sin \theta_{i}\right] \omega^{2} \\
& \left.+\Pi_{0} \frac{\alpha_{i}}{k_{\perp}} k_{z}^{4} v_{A}^{2}\left(2-(J+1) \beta_{i \perp}\right) \frac{T_{e}}{T_{i}} \frac{T_{i z} R_{i}}{m_{i}} \sin \theta_{i}\right\}
\end{aligned}
$$

$$
\begin{aligned}
& \times\left[\left(1+\frac{m_{e}}{m_{i}} \alpha_{i}\right) \frac{5(J+1)^{2}\left(\frac{v_{t i \perp}^{2}}{v_{t i z}^{2}}\right)^{2} \omega^{4}}{k_{z} R_{i}^{2}}\right. \\
& \times \sqrt{\frac{\pi m_{e}}{4 T_{e z} R_{e}}} \cos \left(2 \theta_{i}-\frac{\theta_{e}}{2}\right) \\
& -\left(1+\frac{m_{e}}{m_{i}} \alpha_{i}\right) \frac{4(J+1)^{2}\left(\frac{v_{t i \perp}^{2}}{v_{t i z}^{2}}\right)^{2} \omega^{3}}{R_{i}^{2}} \sin 2 \theta_{i} \\
& -\left(1+\frac{m_{e}}{m_{i}} \alpha_{i}\right) \frac{3 \omega^{2}}{k_{z}} \sqrt{\frac{\pi m_{e}}{4 T_{e z} R_{e}} k_{z}^{2} v_{A}^{2}} \\
& \times\left(1-\frac{\alpha_{i} \beta_{i \perp}(J+1)}{2}\right) \cos \frac{\theta_{e}}{2} \\
& -2 \omega \Pi_{0}\left(1-\alpha_{i}\right) \frac{2(J+1)^{2}\left(\frac{v_{t i \perp}^{2}}{v_{t i z}^{2}}\right)^{2} k_{z}^{2}}{R_{i}} \\
& \left.\times\left(\frac{T_{e}}{T_{i}}\right) \frac{T_{i z}}{m_{i}} \sin \theta_{i}^{-1}\right]^{-1}
\end{aligned}
$$

Using Eqs. (25), (28) and (29) we obtain growth length as

$$
\begin{aligned}
& L_{g}=\left\{-\left[\left(\frac{m_{e}}{m_{i}} \frac{\alpha_{i}}{k_{\perp}}\right) \frac{(J+1)^{2}\left(\frac{v_{t i \perp}^{2}}{v_{t i z}}\right)^{2}}{k_{z} R_{i}^{2}}\right.\right. \\
& \left.\times \sqrt{\frac{\pi m_{e}}{4 T_{e z} R_{e}}} \cos \left(2 \theta_{i}-\frac{\theta_{e}}{2}\right)\right] \omega^{5} \\
& +\left[\left(\frac{m_{e}}{m_{i}} \frac{\alpha_{i}}{k_{\perp}}\right) \frac{(J+1)^{2}\left(\frac{v_{t i \perp}^{2}}{v_{t i z}^{2}}\right)^{2}}{R_{i}^{2}} \sin 2 \theta_{i}\right] \omega^{4} \\
& +\left[\frac{\alpha_{i}}{k_{\perp}} \frac{k_{z}^{2} v_{A}^{2}}{k_{z}}\left(\frac{m_{e}}{m_{i}}-\frac{\beta_{i \perp}(J+1)}{2}\left(1+\frac{2 m_{e}}{m_{i}}\right)\right)\right. \\
& \left.\times \sqrt{\frac{\pi m_{e}}{4 T_{e z} R_{e}}} \cos \frac{\theta_{e}}{2}\right] \omega^{3} \\
& -\left[\Pi_{0} \frac{\alpha_{i}}{k_{\perp}} \frac{k_{z}^{2}(J+1)^{2}\left(\frac{v_{t i \perp}^{2}}{v_{t i z}^{2}}\right)^{2}}{R_{i}}\left(\frac{T_{e}}{T_{i}}\right) \frac{2 T_{i z}}{m_{i}} \sin \theta_{i}\right] \omega^{2} \\
& \left.+\Pi_{0} \frac{\alpha_{i}}{k_{\perp}} k_{z}^{4} v_{A}^{2}\left(2-(J+1) \beta_{i \perp}\right) \frac{T_{e}}{T_{i}} \frac{T_{i z} R_{i}}{m_{i}} \sin \theta_{i}\right\} \\
& \times\left\{-\left[\left(1+\frac{m_{e}}{m_{i}} \alpha_{i}\right) \frac{(J+1)^{2}\left(\frac{v_{t i \perp}^{2}}{v_{t i z}^{2}}\right)^{2}}{k_{z} R_{i}^{2}}\right.\right. \\
& \left.\times \sqrt{\frac{\pi m_{e}}{4 T_{e z} R_{e}}} \sin \left(2 \theta_{i}-\frac{\theta_{e}}{2}\right)\right] \omega^{5} \\
& +\left[\left(1+\frac{m_{e}}{m_{i}} \alpha_{i}\right) \frac{(J+1)^{2}\left(\frac{v_{t i \perp}^{2}}{v_{t i z}^{2}}\right)^{2}}{R_{i}^{2}} \cos 2 \theta_{i}\right] \omega^{4}
\end{aligned}
$$




$$
\begin{aligned}
& -\left[\left(1+\frac{m_{e}}{m_{i}} \alpha_{i}\right) \frac{k_{z}^{2} v_{A}^{2}}{k_{z}}\left(1-\frac{\alpha_{i} \beta_{i \perp}(J+1)}{2}\right)\right. \\
& \left.\times \sqrt{\frac{\pi m_{e}}{4 T_{e z} R_{e}}} \sin \frac{\theta_{e}}{2}\right] \omega^{3} \\
& -\left[\left(1+\frac{m_{e}}{m_{i}} \alpha_{i}\right) k_{z}^{2} v_{A}^{2}\left(1-\frac{\alpha_{i} \beta_{i \perp}(J+1)}{2}\right)\right] \omega^{2} \\
& -\left[\Pi_{0}\left(1-\alpha_{i}\right) \frac{k_{z}^{2}(J+1)^{2}\left(\frac{v_{t i \perp}^{2}}{v_{t i z}^{2}}\right)^{2}}{R_{i}}\left(\frac{T_{e}}{T_{i}}\right) \frac{2 T_{i z}}{m_{i}} \cos \theta_{i}\right. \\
& \left.-\left(\frac{T_{e}}{T_{i}}\right)(J+1) \lambda_{i} k_{z}^{2} v_{A}^{2}\right] \omega^{2} \\
& +\Pi_{0}\left(1-\alpha_{i}\right) k_{z}^{4} v_{A}^{2}\left(1-\frac{\alpha_{i} \beta_{i \perp}(J+1)}{2}\right) \\
& \left.\times \frac{T_{e}}{T_{i}} \frac{2 T_{i z} R_{i}}{m_{i}} \cos \theta_{i}\right\}^{-1}
\end{aligned}
$$

\subsection{Case of strong shear}

The growth length for the KAW driven by strong shear is derived from

$$
L_{g}=\frac{v_{g}}{\gamma}
$$

Using Eq. (23), we derive Group velocity $v_{g}$ as

$$
\begin{aligned}
& v_{g}=\left[-\left(1+\frac{\alpha_{e}}{k_{\perp}}\right) \frac{(J+1)^{2}\left(\frac{v_{t i \perp}^{2}}{v_{t i z}^{2}}\right)^{2} \omega^{5}}{k_{z} R_{i}^{2}}\right. \\
& \times \sqrt{\frac{\pi m_{e}}{4 T_{e z} R_{e}}} \cos \left(2 \theta_{i}-\frac{\theta_{e}}{2}\right) \\
& +\left(1+\frac{\alpha_{e}}{k_{\perp}}\right) \frac{(J+1)^{2}\left(\frac{v_{t i \perp}^{2}}{v_{t i z}}\right)^{2} \omega^{4}}{R_{i}^{2}} \sin 2 \theta_{i} \\
& +\left(1+\frac{\alpha_{e}}{k_{\perp}}\right) \frac{\omega^{3}}{k_{z}} \sqrt{\frac{\pi m_{e}}{4 T_{e z} R_{e}}} k_{z}^{2} v_{A}^{2} \cos \frac{\theta_{e}}{2} \\
& +\frac{\omega^{3}}{k_{z}} \sqrt{\frac{\pi m_{e}}{4 T_{e z} R_{e}}} k_{z}^{2} v_{A}^{2} \frac{m_{i}}{m_{e}} \frac{\alpha_{e} \beta_{i \perp}(J+1)}{2 k_{\perp}}\left(1+2 \alpha_{e}\right) \cos \frac{\theta_{e}}{2} \\
& +\left\{\left(1-\frac{2 \lambda_{i}}{k_{\perp}}\right)\left(1+\frac{m_{i}}{m_{e}} \alpha_{e}\right)+\frac{m_{i}}{m_{e}} \frac{\alpha_{e}}{k_{\perp}} \Pi_{0}\right\} \\
& \times \frac{2 \omega^{2}(J+1)^{2}\left(\frac{v_{t i \perp}^{2}}{v_{t i z}^{2}}\right)^{2} k_{z}^{2}}{R_{i}}\left(\frac{T_{e}}{T_{i}}\right) \frac{T_{i z}}{m_{i}} \sin \theta_{i} \\
& +\Pi_{0} \frac{m_{i}}{m_{e}} \frac{\alpha_{e}}{k_{\perp}}\left\{\left(1+\frac{m_{i}}{m_{e}} \alpha_{e}\right) \frac{\beta_{i \perp}(J+1)}{2}\right. \\
& \left.+\left(1+\frac{m_{i}}{m_{e}} \frac{\alpha_{e} \beta_{i \perp}(J+1)}{2}\right)\right\} \\
& \left.+\left(1-\frac{2 \lambda_{i}}{k_{\perp}}\right)\left(1+\frac{m_{i}}{m_{e}} \alpha_{e}\right)\left(1+\frac{m_{i}}{m_{e}} \frac{\alpha_{e} \beta_{i \perp}(J+1)}{2}\right)\right] \\
& \times\left\{5 \left[\left(1+\alpha_{e}\right) \frac{(J+1)^{2}\left(\frac{v_{t i \perp}^{2}}{v_{t i z}^{2}}\right)^{2}}{k_{z} R_{i}^{2}}\right.\right.
\end{aligned}
$$

$$
\begin{aligned}
& \left.\times \sqrt{\frac{\pi m_{e}}{4 T_{e z} R_{e}}} \cos \left(2 \theta_{i}-\frac{\theta_{e}}{2}\right)\right] \omega^{4} \\
& -4\left[\left(1+\alpha_{e}\right) \frac{(J+1)^{2}\left(\frac{v_{t i \perp}^{2}}{v_{t i z}^{2}}\right)^{2}}{R_{i}^{2}} \sin 2 \theta_{i}\right] \omega^{3} \\
& -3\left[\left(1+\alpha_{e}\right)+\frac{m_{i}}{m_{e}} \frac{\alpha_{e} \beta_{i \perp}(J+1)}{2}\right] \\
& \times \frac{k_{z}^{2} v_{A}^{2}}{k_{z}} \sqrt{\frac{\pi m_{e}}{4 T_{e z} R_{e}}} \cos \frac{\theta_{e}}{2} \omega^{2} \\
& -2\left[\Pi_{0}\left(1+\frac{m_{i}}{m_{e}} \alpha_{e}\right) \frac{k_{z}^{2}(J+1)^{2}\left(\frac{v_{t i \perp}^{2}}{v_{t i z}^{2}}\right)^{2}}{R_{i}}\right. \\
& \left.\left.\times\left(\frac{T_{e}}{T_{i}}\right) \frac{2 T_{i z}}{m_{i}} \sin \theta_{i}\right]\left.\right|^{-1}\right\}^{-1}
\end{aligned}
$$

Using Eqs. (27), (31) and (32), we obtain growth length as

$$
\begin{aligned}
& L_{g}=\left[-\left(1+\frac{\alpha_{e}}{k_{\perp}}\right) \frac{(J+1)^{2}\left(\frac{v_{t i \perp}^{2}}{v_{t i z}^{2}}\right)^{2} \omega^{5}}{k_{z} R_{i}^{2}}\right. \\
& \times \sqrt{\frac{\pi m_{e}}{4 T_{e z} R_{e}}} \cos \left(2 \theta_{i}-\frac{\theta_{e}}{2}\right) \\
& +\left(1+\frac{\alpha_{e}}{k_{\perp}}\right) \frac{(J+1)^{2}\left(\frac{v_{t i \perp}^{2}}{v_{t i z}^{2}}\right)^{2} \omega^{4}}{R_{i}^{2}} \sin 2 \theta_{i} \\
& +\left(1+\frac{\alpha_{e}}{k_{\perp}}\right) \frac{\omega^{3}}{k_{z}} \sqrt{\frac{\pi m_{e}}{4 T_{e z} R_{e}}} k_{z}^{2} v_{A}^{2} \cos \frac{\theta_{e}}{2} \\
& +\frac{\omega^{3}}{k_{z}} \sqrt{\frac{\pi m_{e}}{4 T_{e z} R_{e}}} k_{z}^{2} v_{A}^{2} \frac{m_{i}}{m_{e}} \frac{\alpha_{e} \beta_{i \perp}(J+1)}{2 k_{\perp}}\left(1+2 \alpha_{e}\right) \cos \frac{\theta_{e}}{2} \\
& +\left\{\left(1-\frac{2 \lambda_{i}}{k_{\perp}}\right)\left(1+\frac{m_{i}}{m_{e}} \alpha_{e}\right)+\frac{m_{i}}{m_{e}} \frac{\alpha_{e}}{k_{\perp}} \Pi_{0}\right\} \\
& \times \frac{2 \omega^{2}(J+1)^{2}\left(\frac{v_{t i \perp}^{2}}{v_{t i z}^{2}}\right)^{2} k_{z}^{2}}{R_{i}}\left(\frac{T_{e}}{T_{i}}\right) \frac{T_{i z}}{m_{i}} \sin \theta_{i} \\
& +\Pi_{0} \frac{m_{i}}{m_{e}} \frac{\alpha_{e}}{k_{\perp}}\left\{\left(1+\frac{m_{i}}{m_{e}} \alpha_{e}\right) \frac{\beta_{i \perp}(J+1)}{2}\right. \\
& \left.+\left(1+\frac{m_{i}}{m_{e}} \frac{\alpha_{e} \beta_{i \perp}(J+1)}{2}\right)\right\} \\
& \left.+\left(1-\frac{2 \lambda_{i}}{k_{\perp}}\right)\left(1+\frac{m_{i}}{m_{e}} \alpha_{e}\right)\left(1+\frac{m_{i}}{m_{e}} \frac{\alpha_{e} \beta_{i \perp}(J+1)}{2}\right)\right] \\
& \times\left[-\left(1+\alpha_{e}\right) \frac{(J+1)^{2}\left(\frac{v_{t i \perp}^{2}}{v_{t i z}^{2}}\right)^{2} \omega^{5}}{k_{z} R_{i}^{2}}\right. \\
& \times \sqrt{\frac{\pi m_{e}}{4 T_{e z} R_{e}}} \cos \left(2 \theta_{i}-\frac{\theta_{e}}{2}\right) \\
& +\left(1+\alpha_{e}\right) \frac{(J+1)^{2}\left(\frac{v_{t i \perp}^{2}}{v_{t i z}^{2}}\right)^{2} \omega^{4}}{R_{i}^{2}} \sin 2 \theta
\end{aligned}
$$




$$
\begin{aligned}
& +\left(1+\alpha_{e}\right) \frac{\omega^{3}}{k_{z}} \sqrt{\frac{\pi m_{e}}{4 T_{e z} R_{e}}} k_{z}^{2} v_{A}^{2}\left(1-\frac{\alpha_{i} \beta_{i \perp}(J+1)}{2}\right) \cos \frac{\theta_{e}}{2} \\
& +\omega^{2} \Pi_{0}\left(1-\alpha_{i}\right) \frac{2(J+1)^{2}\left(\frac{v_{t i \perp}^{2}}{v_{t i z}^{2}}\right)^{2} k_{z}^{2}}{R_{i}}\left(\frac{T_{e}}{T_{i}}\right) \frac{T_{i z}}{m_{i}} \sin \theta_{i} \\
& +\Pi_{0}\left(1-\alpha_{i}\right) k_{z}^{2}\left(\frac{T_{e}}{T_{i}}\right) \frac{2 T_{i z} R_{i}}{m_{i}} k_{z}^{2} v_{A}^{2} \\
& \left.\times\left(1-\frac{\alpha_{i} \beta_{i \perp}(J+1)}{2}\right) \sin \theta_{i}\right]^{-1}
\end{aligned}
$$

\section{Result and Discussion}

A graphical analysis of the results is shown in Figs. 112. Parameters appropriate to the PSBL region are used (Wygant et al., 2000, 2002; Scime et al., 2003; Keling, 2005).

$$
\begin{aligned}
& B_{0}=400 \mathrm{nT}, \quad \omega_{c i}=38.33 \mathrm{~Hz}, \quad n_{0}=1 \mathrm{~cm}^{-3}, \\
& v_{A}=8720 \mathrm{~km} / \mathrm{s}, \quad T_{\perp e}=25-50 \mathrm{eV}, \quad T_{z e}=2 \mathrm{keV}, \\
& T_{\perp i}=4 \mathrm{keV}, \quad T_{z i}=1-2 \mathrm{keV}, \quad \frac{T_{e}}{T_{i}}=0.5 \mathrm{keV}, \\
& \rho_{c i}=2.285 \times 10^{6} \mathrm{~cm}=22.85 \mathrm{~km}, \\
& k_{z}=1 \times 10^{-9} \mathrm{~cm}^{-1}, \quad V_{0}=400 \mathrm{~km} / \mathrm{s} .
\end{aligned}
$$

Figures 1-6 show the effect of the loss-cone distribution function, parallel electric field and ion temperature anisotropy on the frequency and growth length of KAW in the weak shear regime $(\beta v=0.04$ and 0.06$)$.

Figure 1 shows the variation in wave frequency $\left(\frac{\omega}{\omega_{c i}}\right)$ with the perpendicular wave vector $\left(k_{\perp} \rho_{c i}\right)$ for different values of the loss-cone distribution index $(J)$ in the weak shear regime $(\beta v)$ with $E_{0}=10 \mathrm{mV} / \mathrm{m}$. It is observed that for $J=0$-i.e. for the bi-Maxwellian distribution functionthe wave frequency enhances with the perpendicular wave number, which is characteristic of KAW. The effect of the loss-cone distribution index $(J=1,2)$ is to decrease the wave frequency, and it is less prominent for the lower values of $k_{\perp} \rho_{c i}$. As $k_{\perp} \rho_{c i}$ approaches 1 , a steep decrease in the wave frequency is observed. The higher distribution indices decrease the frequency bandwidth towards lower $k_{\perp} \rho_{c i}$. For $J=2$, the frequency even becomes negative, representing the reflection of the wave. This may be due to the mirroring effect in the loss-cone distribution at higher $k_{\perp} \rho_{c i}$. The decrease in the frequency of KAW due to the loss-cone distribution index $(J)$ may be due to the decrease in the ion drift velocity by the averaging of wave field over the Larmor orbit in the presence of the steep loss-cone distribution function. The steepness (higher $J$ ) of the loss-cone distribution index becomes apparent through the ion gyroradius effects, which actually determine the wave frequency. The electron Larmor radius effect does not significantly contribute to the wave frequency because $k_{\perp} \rho_{c e} \ll 1$, so that the wave frequency is not affected by the value of the losscone distribution index $(J)$ due to the small effect of the electron gyro-radius. Thus, a significant effect may appear in the wave frequency by the finite ion Larmor radius effect. The spatial scales of the perpendicular perturbation driving KAWs have a tendency to decrease with large values of $J$,

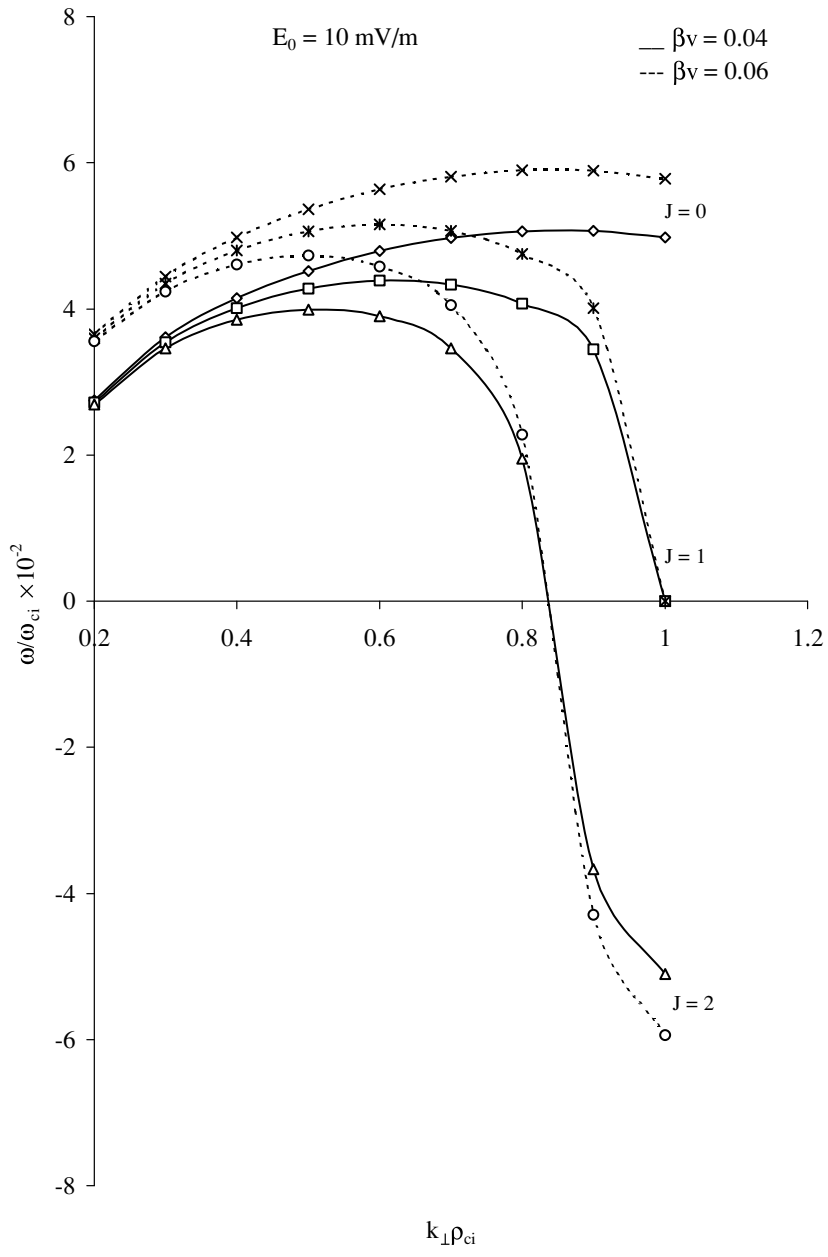

Fig. 1. Variation in wave frequency $\left(\frac{\omega}{\omega_{c i}}\right)$ with perpendicular wave number $\left(k_{\perp} \rho_{c i}\right)$ for different values of the loss-cone distribution index $(J)$ and weak shear $(\beta v)$ with $E_{0}=10 \mathrm{mV} / \mathrm{m}$.

which is perhaps in relation with the decreasing width of the loss-cone. The observed effects of the loss-cone distribution index $(J)$ on KAW driven by shear are also similar to those obtained by Duan et al. (2005) in the absence of the shear. It is further observed that the increasing values of the weak shear enhance the frequency of the KAW. Thus, KAWs with enhanced frequencies due to increasing values of weak shear may be observed at the onset of the substorm. The observed frequency of the KAW in the present study is in agreement with the reported values of $0.1-4 \mathrm{~Hz}$ in the PSBL region (Wygant et al., 2002; Keiling et al., 2005).

Figure 2 shows the variation in growth length $\left(L_{g}\right)$ with the perpendicular wave number $\left(k_{\perp} \rho_{c i}\right)$ for different values of the loss-cone distribution index $(J)$ in the weak shear regime $(\beta v)$ with $E_{0}=10 \mathrm{mV} / \mathrm{m}$. It is observed that for $J=0$-i.e. for the bi-Maxwellian distribution of particles - the growth length decreases with $k_{\perp} \rho_{c i}$ and is at its minimum at $k_{\perp} \rho_{c i} \sim 1$. This may be due to the cyclotron interaction of the KAW with the plasma particles. It is further observed that for the loss-cone distribution of particles $(J=1)$ the growth length $\left(L_{g}\right)$ decreases with $k_{\perp} \rho_{c i}$, attains a peak and then decreases again. The peak in the growth length represents the resonance condition of the wave-particle interaction. A shift in the peak is observed 


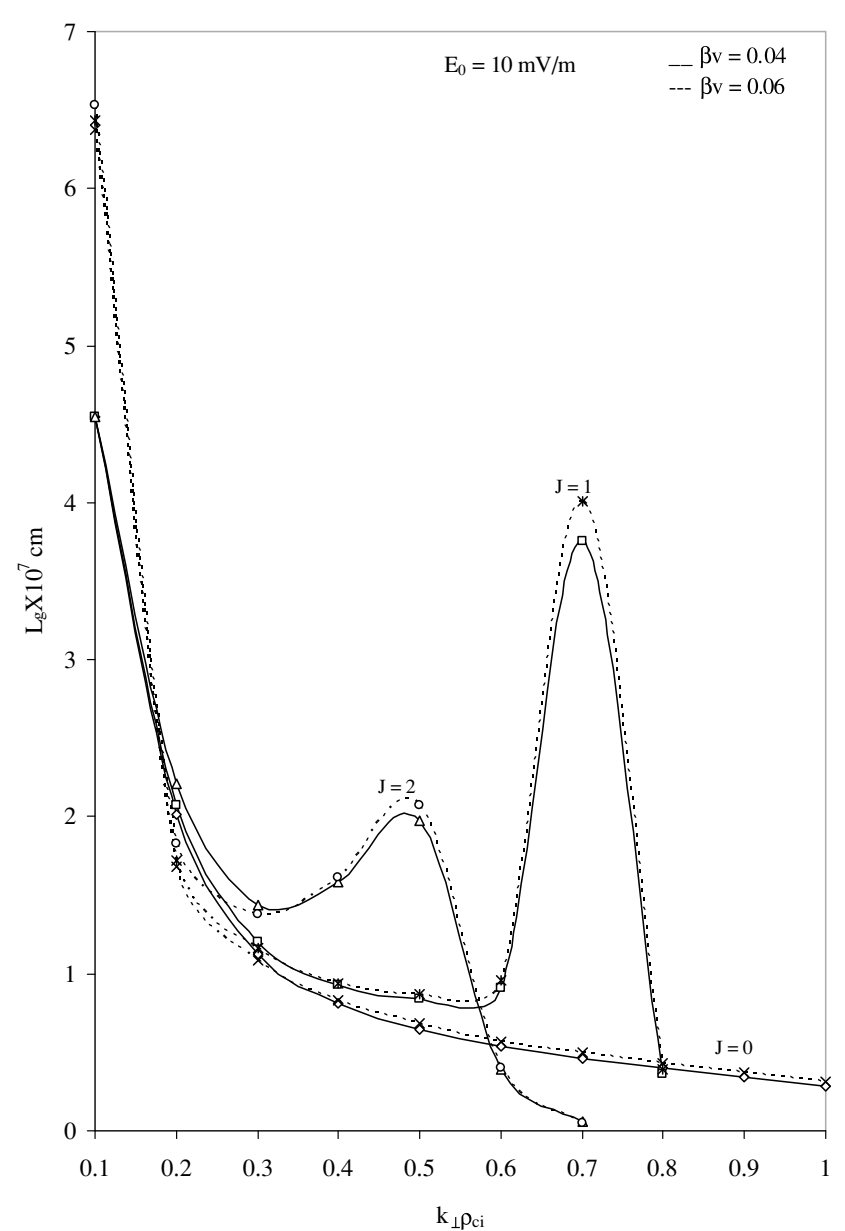

Fig. 2. Variation in growth length $\left(L_{g}\right)$ with perpendicular wave number $\left(k_{\perp} \rho_{c i}\right)$ for different values of the loss-cone distribution index $(J)$ and weak shear $(\beta v)$ with $E_{0}=10 \mathrm{mV} / \mathrm{m}$.

towards the lower side of $k_{\perp} \rho_{c i}$ by the enhancement in the loss-cone distribution index. For $J=2$, the nature of the growth length remains the same as that for $J=1$, but the peak value shifts towards the lower side of $k_{\perp} \rho_{c i}$ and the maximum value of growth length attained also becomes smaller, possibly in relation with the decreasing width of the loss-cone (Summers and Thorne, 1995). The steep losscone structures are analogous to mirror-like devices with a higher mirror ratio that may accelerate the charged particles moving perpendicular to the magnetic field. Thus, more energetic particles may be available to provide energy to the wave by resonance wave-particle interaction. Further, the effect of the increasing values of weak shear $(\beta v)$ is to slightly enhance the growth length. This may be due to the cyclotron interaction of the KAW with the plasma particles.

Figure 3 shows the variation in wave frequency $\left(\frac{\omega}{\omega_{c i}}\right)$ with perpendicular wave number $\left(k_{\perp} \rho_{c i}\right)$ for different values of the parallel electric field $\left(E_{0}\right)$ with the loss-cone distribution index $(J=1)$. It is observed that the frequency of the KAW in the presence of the loss-cone distribution index $(J=1)$ enhances with $k_{\perp} \rho_{c i}$, which is in accordance with the characteristic of KAW, followed by a steep decrease near $k_{\perp} \rho_{c i} \sim 1$, which may be due to the mirroring effect of the loss-cone, as discussed earlier. It is also observed that

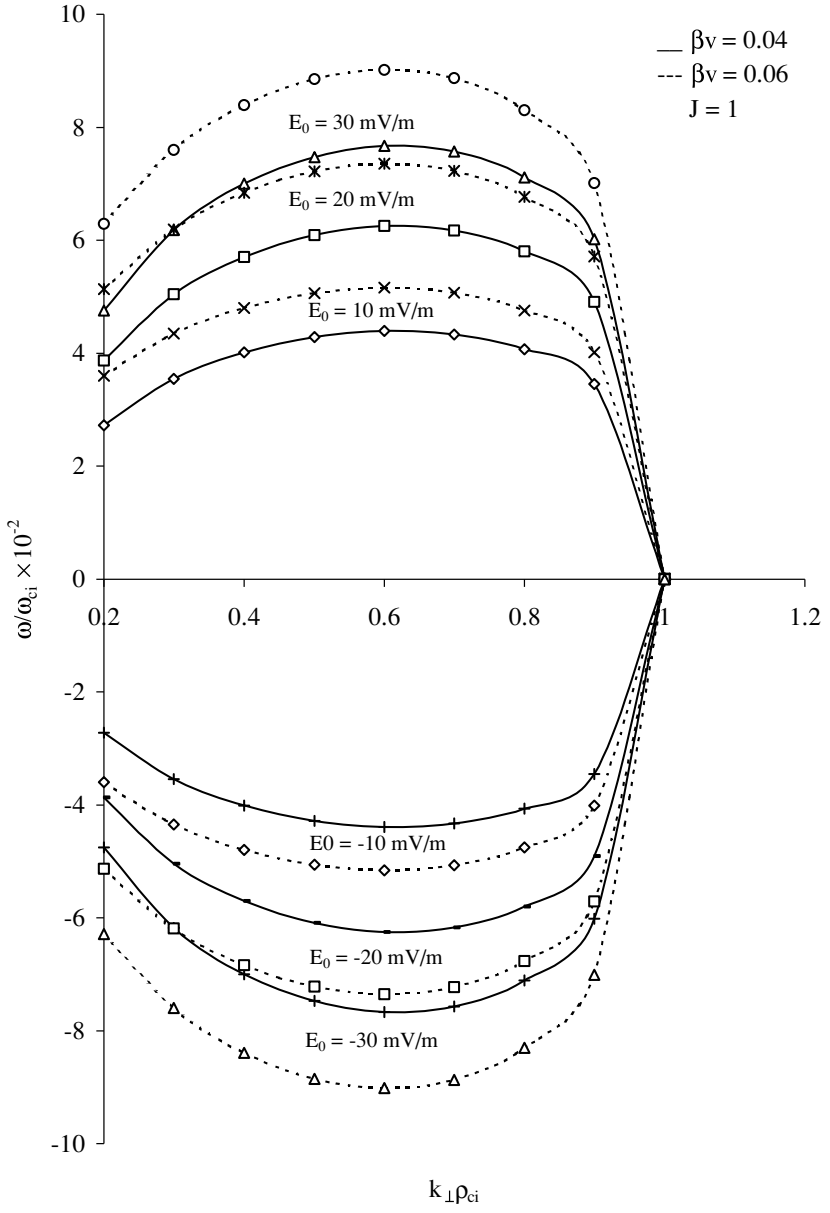

Fig. 3. Variation in wave frequency $\left(\frac{\omega}{\omega_{c i}}\right)$ with perpendicular wave number $\left(k_{\perp} \rho_{c i}\right)$ for different values of parallel electric field $\left(E_{0}\right)$ and weak shear $(\beta v)$ with $J=1$.

for a fixed value of weak shear $(\beta v)$, the effect of the parallel electric field is to enhance the wave frequency, suggesting that the Alfven wave propagates from the PSBL to the ionosphere at higher frequencies. This is consistent with the observed characteristic of the KAWs (Keiling et al., 2005). Keiling et al. (2005) recently analysed the KAWs observed by the Polar satellite in the PSBL and reported that the peak $E$ to $B$ ratio (i.e. Alfven speed) for the highest frequency waves was larger than that for the lowest frequency waves, which is consistent with a transition towards kinetic Alfven waves. It is further observed that the negative values of parallel electric fields (fields directed towards the magnetotail) give a negative frequency to the wave, implying that the tailward-directed electric fields give rise to the reflected Alfven waves with a frequency almost the same as that of the incident waves. This is in agreement with the results of Vogt and Harrendel (1998). The effect of the increasing values of tailward-directed electric fields is to enhance the frequency of the reflected wave. Thus, the electric fields in the PSBL and the auroral acceleration region may explain the generation of the recently observed (Keiling et al., 2005) travelling (both incident and reflected) shear-driven KAWs in the PSBL at the onset of the substrom. Figure 3 also shows that the effect of weak shear $(\beta v)$ in the presence of the parallel electric field and loss-cone distribution index 


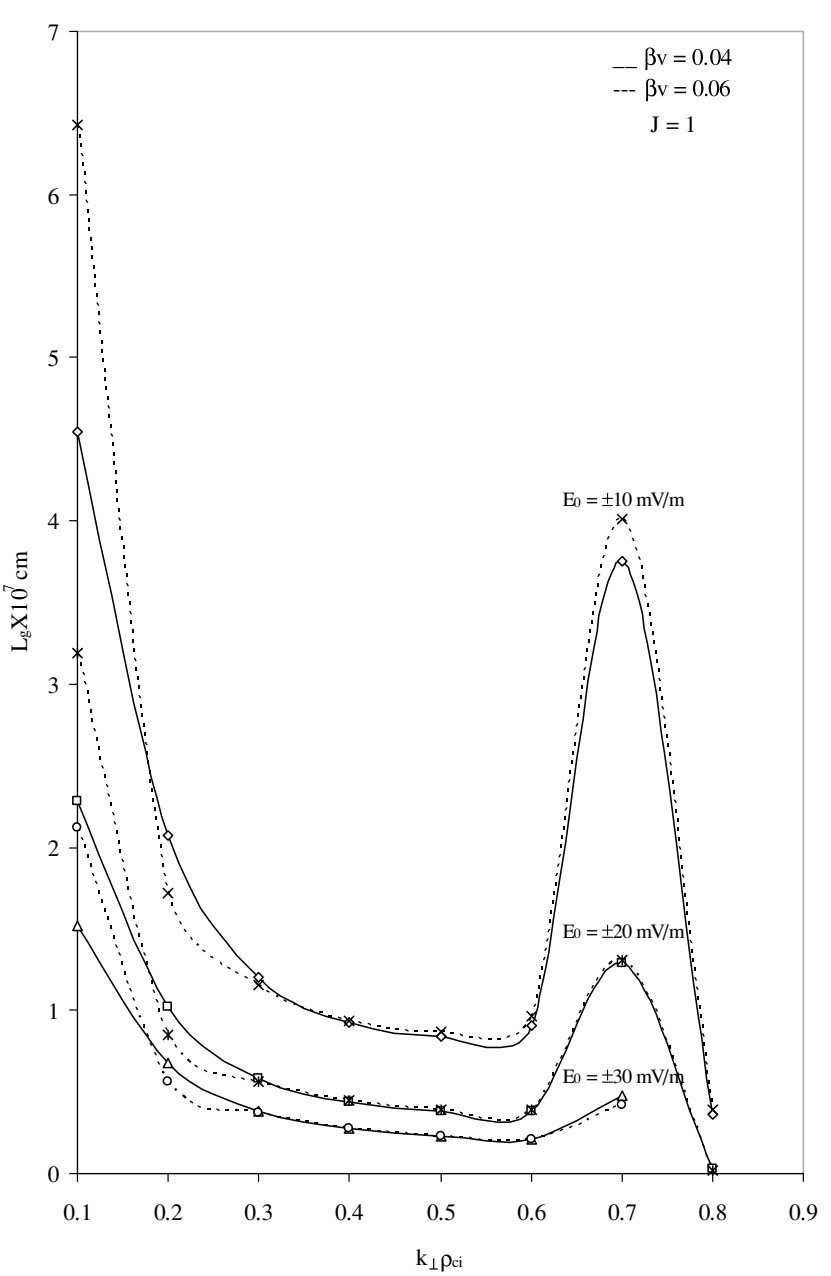

Fig. 4. Variation in growth length $\left(L_{g}\right)$ with perpendicular wave number $\left(k_{\perp} \rho_{c i}\right)$ for different values of parallel electric field $\left(E_{0}\right)$ and weak shear $(\beta v)$ with $J=1$.

is to enhance the frequency of the KAW.

Figure 4 shows the variation in the growth length $\left(L_{g}\right)$ with perpendicular wave number $\left(k_{\perp} \rho_{c i}\right)$ for different values of the parallel electric field $\left(E_{0}\right)$ in the weak shear regime $(\beta v)$ with $J=1$. It is observed that the growth length decreases with $k_{\perp} \rho_{c i}$ and is at a minimum at $k_{\perp} \rho_{c i} \sim$ 1. A peak in the curve occurs due to the loss-cone distribution index $(J=1)$, implying that the resonance interaction of the KAW with the plasma particles takes place at lower perpendicular perturbations.

It is further observed that the increasing values of the parallel electric field $\left(E_{0}\right)$ decrease the growth length, which may be due to the Landau damping of the wave in the presence of the parallel electric field. Figure 4 also shows that for the tailward (negative)-directed parallel electric field, the growth length attained by the reflected kinetic Alfven wave is same as that of the incident wave. However, Keiling et al. (2005) reported that the Poynting flux carried by these reflected waves $\left(<0.05 \mathrm{erg} / \mathrm{cm}^{2} / \mathrm{s}\right)$ is twofold lower than that carried by the incident waves $\left(>0.1 \mathrm{erg} / \mathrm{cm}^{2} / \mathrm{s}\right)$, implying that a portion of the incident Poynting flux is dissipated somewhere in the auroral acceleration region. The incident and reflected shear-driven KAWs may superimpose. When both the incident and the reflected waves are in phase, they

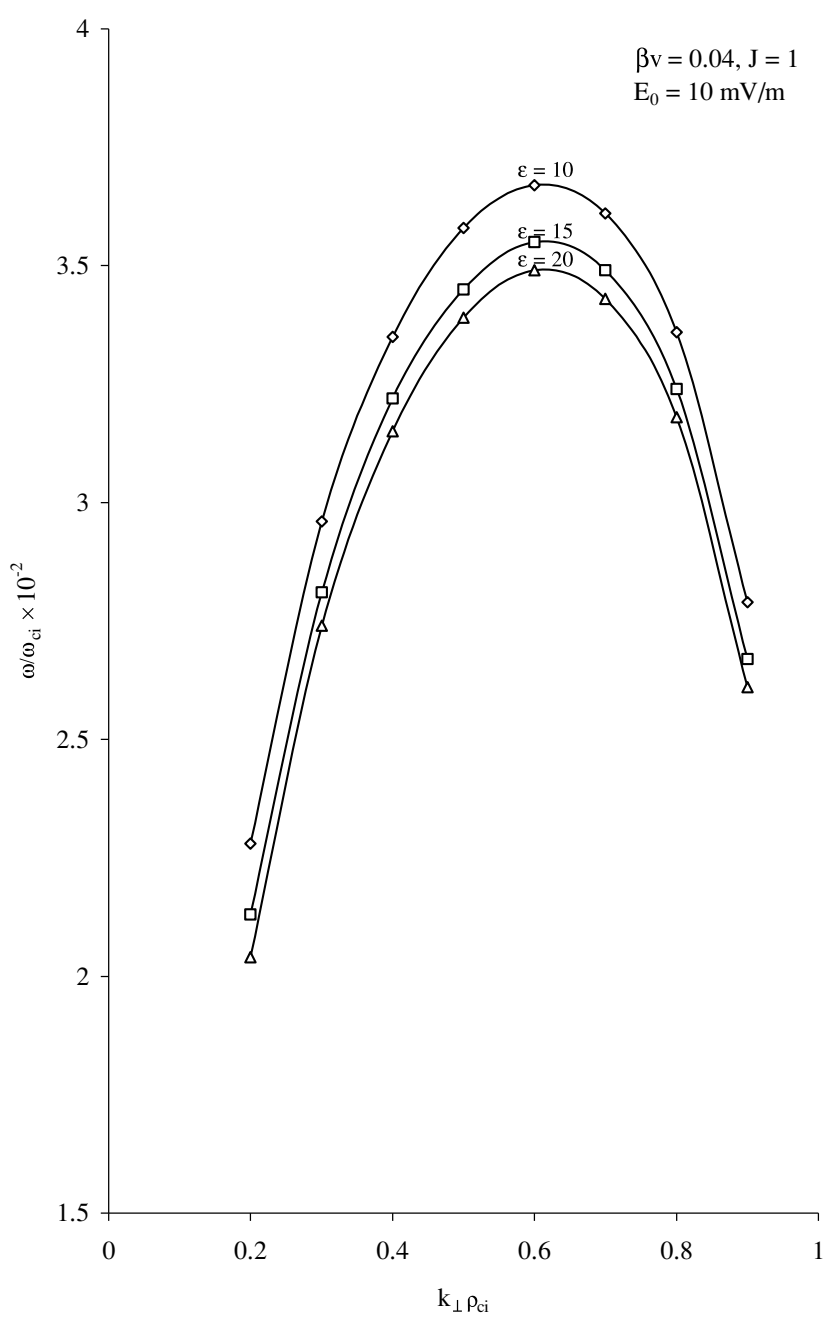

Fig. 5. Variation in wave frequency $\left(\frac{\omega}{\omega_{c i}}\right)$ with perpendicular wave number $\left(k_{\perp} \rho_{c i}\right)$ for different values of ion thermal anisotropy $(\varepsilon)$ with $J=1, \beta v=0.04$ and $E_{0}=10 \mathrm{mV} / \mathrm{m}$.

may superimpose to give the observed (Wygant et al., 2000, 2002; Keiling et al., 2005) large-amplitude, high-frequency shear-driven KAWs, and when out of phase, they may superimpose to give the observed (Keiling et al., 2005) standing waves with a net Poynting flux of nearly zero. These standing waves may be local in nature. In Fig. 4, it is further observed that the effect of increasing values of weak shear $(\beta v)$ is to slightly enhance the growth length. This may be due to the cyclotron interaction of the KAW with plasma particles.

Figures 5 and 6 show the effect of ion thermal anisotropy $\varepsilon=v_{t i \perp}^{2} / v_{t i z}^{2}$ on the frequency $\left(\frac{\omega}{\omega_{c i}}\right)$ and growth length $\left(L_{g}\right)$ of KAW in the weak shear regime $(\beta v=0.04)$ with loss-cone distribution index $(J=1)$ and $E_{0}=10 \mathrm{mV} / \mathrm{m}$. It is observed that the ion thermal anisotropy $(\varepsilon)$ decreases the frequency and reduces the frequency band and growth length of KAW. Thus, the thermal anisotropy of ions in the PSBL may generate KAWs with a narrowed frequency band. The effect of ion thermal anisotropy $(\varepsilon)$ on the sheardriven KAW in the presence of the loss-cone distribution index is similar to that reported by Tiwari et al. (2006). However, the profile of the graph has changed due to the 


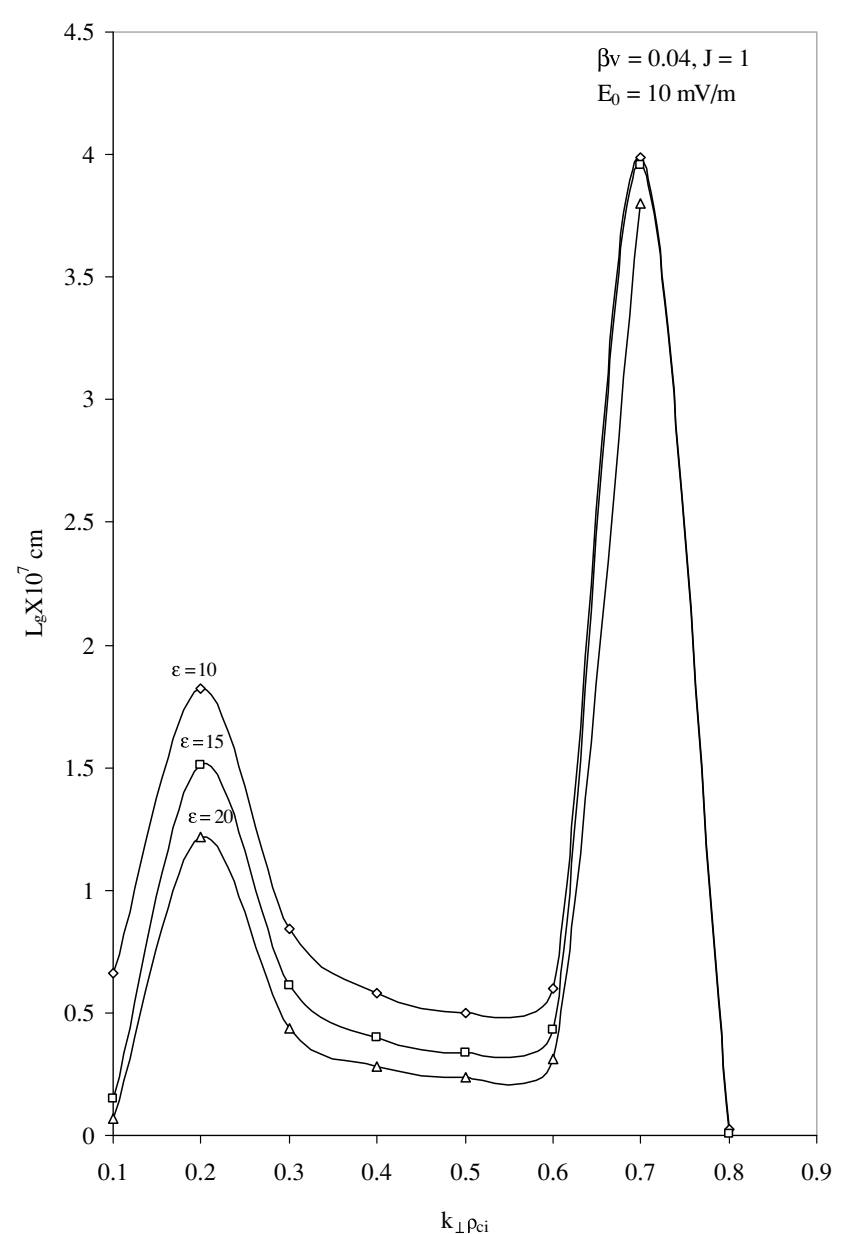

Fig. 6. Variation in growth length $\left(L_{g}\right)$ with perpendicular wave number $\left(k_{\perp} \rho_{c i}\right)$ for different values of ion thermal anisotropy $(\varepsilon)$ with $J=1$, $\beta v=0.04$ and $E_{0}=10 \mathrm{mV} / \mathrm{m}$.

loss-cone distribution index $(J)$.

Thus, at the onset of the substorm, when the velocity shear is weak, energetic KAW may be generated. Largeamplitude KAWs are often observed in the PSBL during substorms (Wygant et al., 2000, 2002; Nakamura et al., 2004; Keiling et al., 2005), and various generation mechanisms have been proposed (e.g. Wygant et al., 2002). Here, we propose a mechanism that these large-amplitude KAWs in the PSBL are generated by the weak shear at the onset of the substorm. The velocity shear acts as a source of free energy and generates the KAW at an enhanced growth rate at the substorm onset. Given the limits of the large obliqueness angles of KAW, this generation is through the interaction of the cyclotron. The observed frequency of the KAW in the present study is in agreement with the reported values of $0.1-4 \mathrm{~Hz}$ in the PSBL region (Wygant et al., 2002). The electric field present along the magnetic field lines may reflect these waves, which may then superimpose in phase or out of phase to give the observed (Keiling et al., 2005) large-amplitude, high-frequency KAWs or the standing waves. The weak shear at the onset may further enhance the growth length of the incident and reflected waves.

At the substorm onset, the loss-cone distribution function enhances the growth length of the KAW. The steep loss-

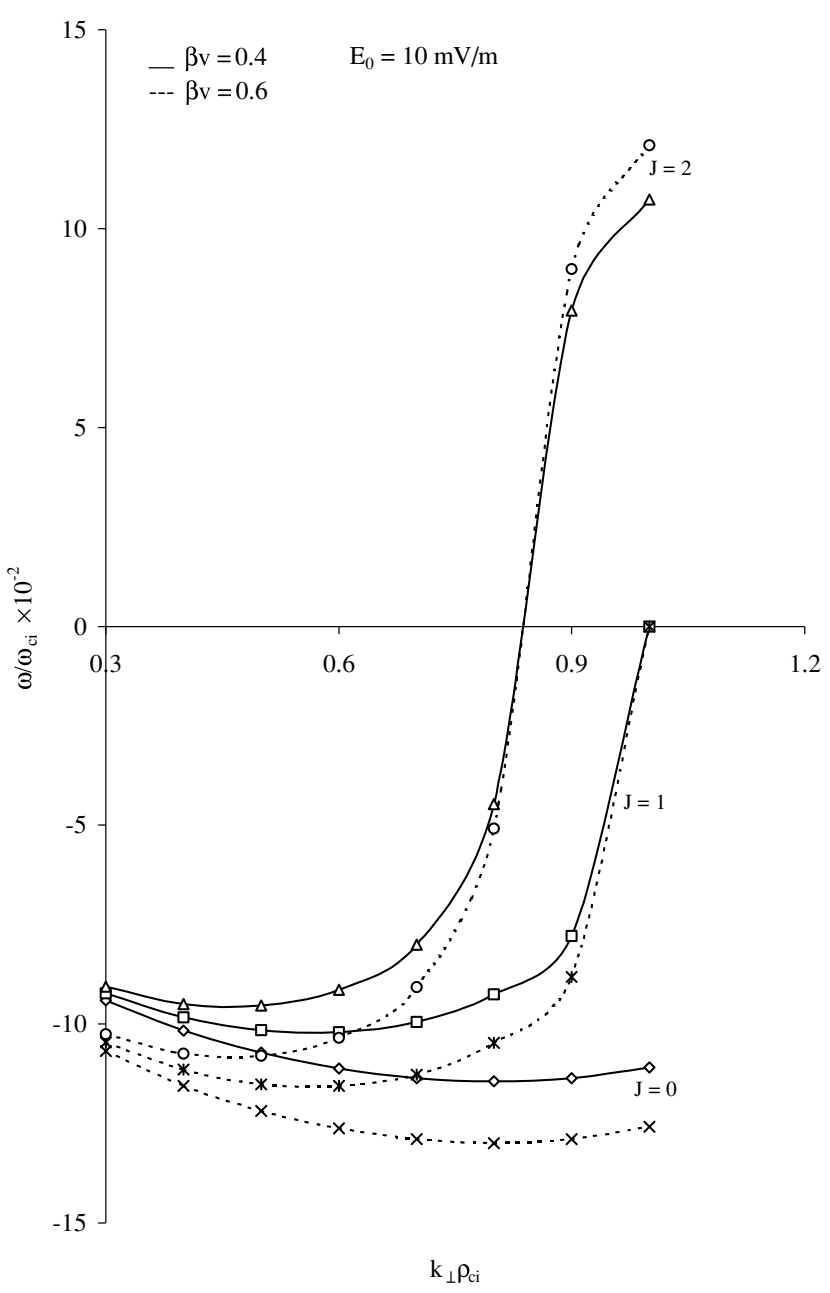

Fig. 7. Variation in wave frequency $\left(\frac{\omega}{\omega_{c i}}\right)$ with perpendicular wave number $\left(k_{\perp} \rho_{c i}\right)$ for different values of loss-cone distribution index $(J)$ and strong shear $(\beta v)$ with $E_{0}=10 \mathrm{mV} / \mathrm{m}$.

cone structures are analogous to mirror-like devices with a higher mirror-ratio that may accelerate the charged particles moving perpendicular to the magnetic field. Hence, a larger number of particles may be available to provide energy to the wave.

Keiling et al. (2005) have reported that the Alfven waves associated with the substorm onset at the PSBL are a mixture of standing and travelling (both earthward and tailward) waves for different frequency ranges. The Alfven wave with the slowest variations (70-300 s) was a travelling wave since $E_{z}$ and $B_{y}$ were in phase. The $E$ to $B$ ratio of the main peak is about $8000 \mathrm{~km} / \mathrm{s}$ and is consistent with the Alfven wave interpretation, and the Poynting flux is mostly downward (Keiling et al., 2005). The next period range (40-67 s) shows standing Alfven waves where $E_{z}$ and $B_{y}$ were in a phase quadrature. The standing wave signature also manifests in the alternating Poynting flux (Keiling et al., 2005). Keiling et al. (2005) have further shown that the next two period ranges (14-24 s and 9.5-12 s) show both earthwardand tailward-travelling Alfven waves. Most of the Poynting flux $\left(>0.1 \mathrm{erg} / \mathrm{cm}^{2} / \mathrm{s}\right)$ was carried in downward-travelling waves in the PSBL (Keiling et al., 2005). The magnitude of the reflected Poynting flux $\left(<0.05 \mathrm{erg} / \mathrm{cm}^{2} / \mathrm{s}\right)$ is twofold 


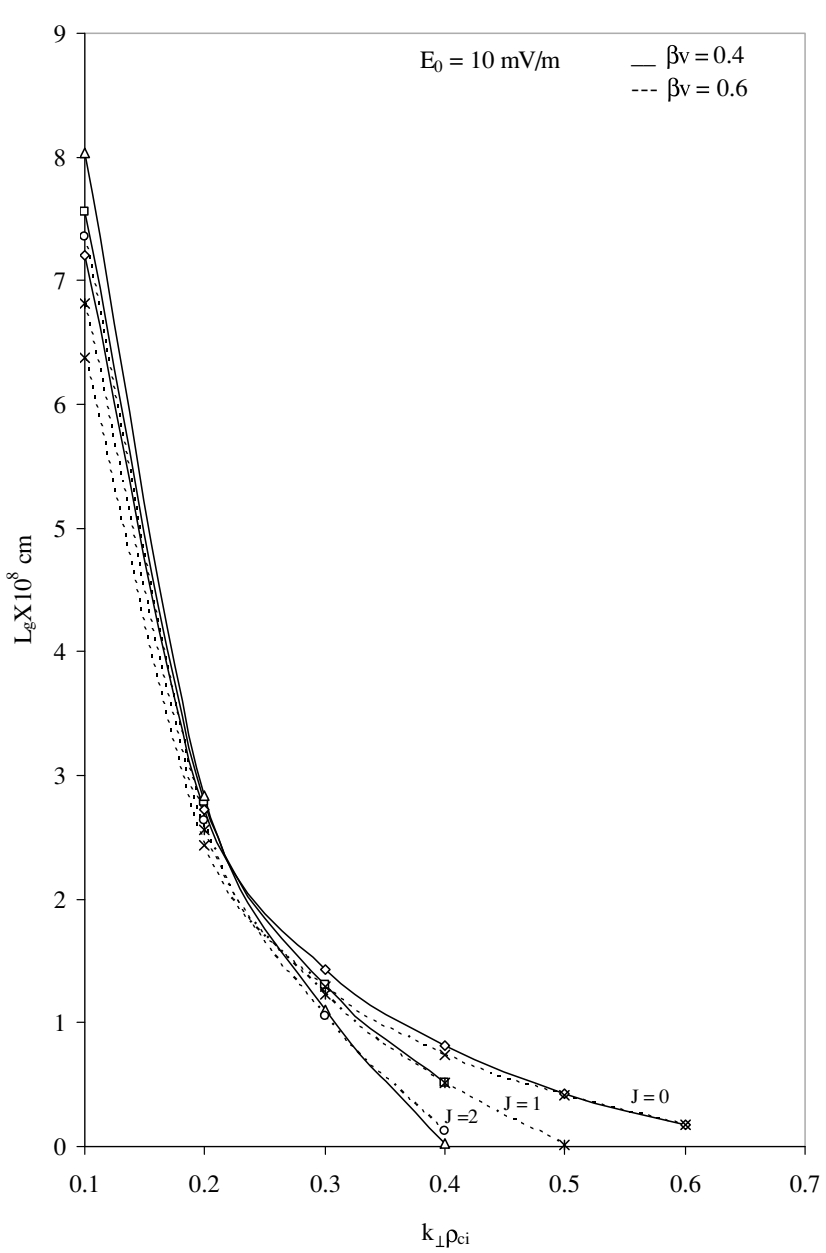

Fig. 8. Variation in growth length $\left(L_{g}\right)$ with perpendicular wave number $\left(k_{\perp} \rho_{c i}\right)$ for different values of loss-cone distribution index $(J)$ and strong shear $(\beta v)$ with $E_{0}=10 \mathrm{mV} / \mathrm{m}$.

less than that of the largest earthward-directed Poynting flux (Keiling et al., 2005).

The results of the present study have shown that at substorm onset, the parallel electric field may reflect the highfrequency, large-amplitude, shear-driven KAWs. Thus, at substorm onset, earthward- and tailward-travelling KAWs may be generated by the weak shear in the presence of the parallel electric field. The importance of the waves reflected from the sheared flow layer has been pointed out by various researchers (e.g. Wu and Seyler, 2003). Wu and Seyler (2003) have shown that since the reflected wave travels away from the shear layer, there is no continuous growth of the wave. On the other hand, if the boundary condition can be altered in some way, then an instability can develop, since the wave would be continuously amplified as it bounces between the shear layer and the reflection boundary. This is analogous to the physics of lasers (Wu and Seyler, 2003).

Wygant et al. $(2000,2002)$ have recently reported their observations of intense field-aligned, earthward-flowing electrons along with the intense small-scale KAW in the PSBL region. The parallel electric field that developed due to a number of factors (Hull et al., 2003) in the presence of KAW at the substorm onset may heat the fast and field-

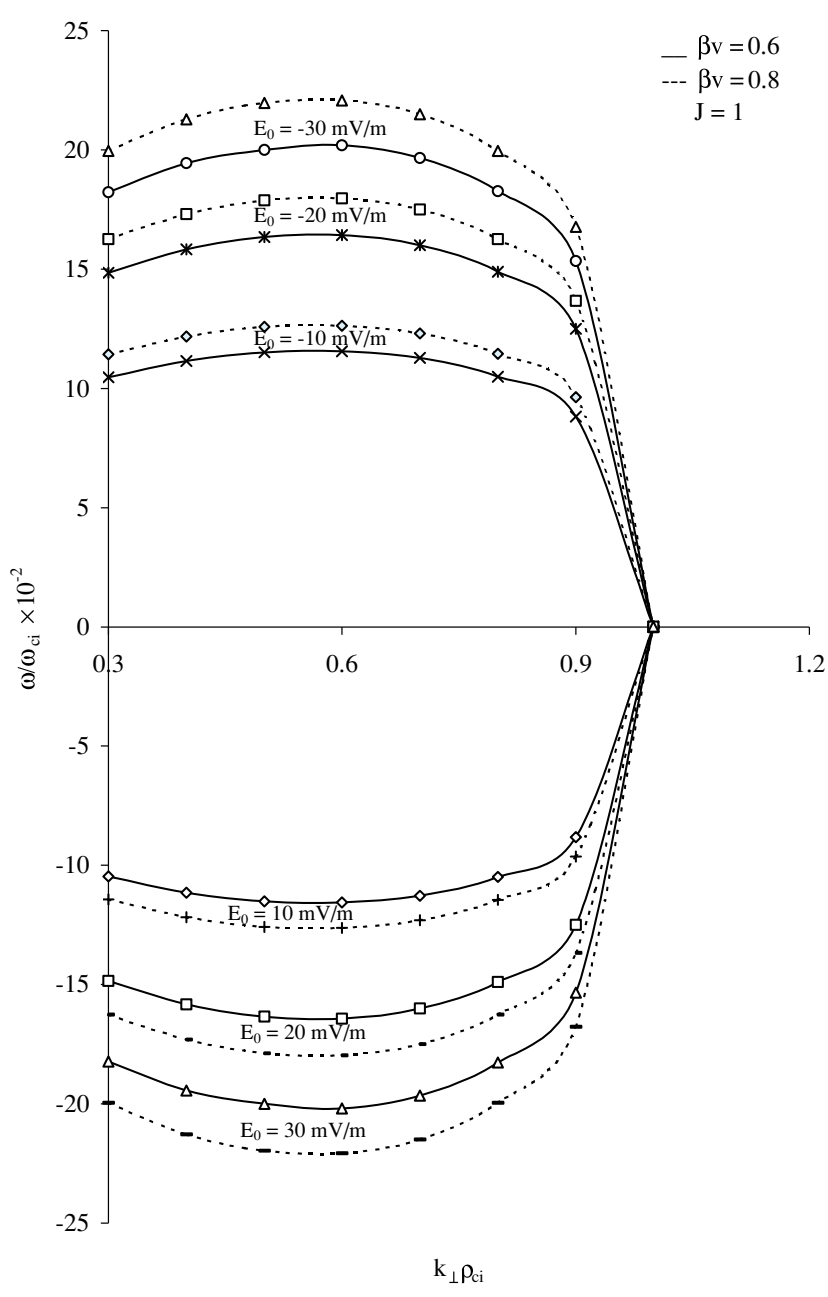

Fig. 9. Variation in wave frequency $\left(\frac{\omega}{\omega_{c i}}\right)$ with perpendicular wave number $\left(k_{\perp} \rho_{c i}\right)$ for different values of parallel electric field $\left(E_{0}\right)$ and strong shear $(\beta v)$ with $J=1$.

aligned electrons interacting with the waves, leading to the parallel electron energisation that ultimately causes an intense aurora. The mechanism is similar to that proposed by Hasegawa and Chen (1975), Wygant et al. (2002) and Angelopoulos et al. (2002). Hasegawa and Chen (1975) initially proposed that when the incoming compressional oscillations reach the resonant layer, they become kinetic, subsequently propagating across the magnetic field because of the finite perpendicular wavelength supported by the finite ion gyroradius and/or the finite electron inertia. In the collisionless regime, the parallel electric field of the KAW supplemented by the external parallel electric field interacts with and heats primarily the electrons. Wygant et al. (2002) have also reported that at altitudes of approximately $5 R_{E}$, the electron thermal velocity is roughly comparable to the Alfven velocity, leading to the possibility of resonant interactions between waves and electrons. KAWs with perpendicular scales of the order of an electron inertial length are often associated with small but significant parallel electric fields extending over a very long distance that are parallel to the magnetic field and can efficiently accelerate charged particles through this electric field (Wygant et al., 2002).

Figures 7-12 show the effects of the loss-cone distri- 


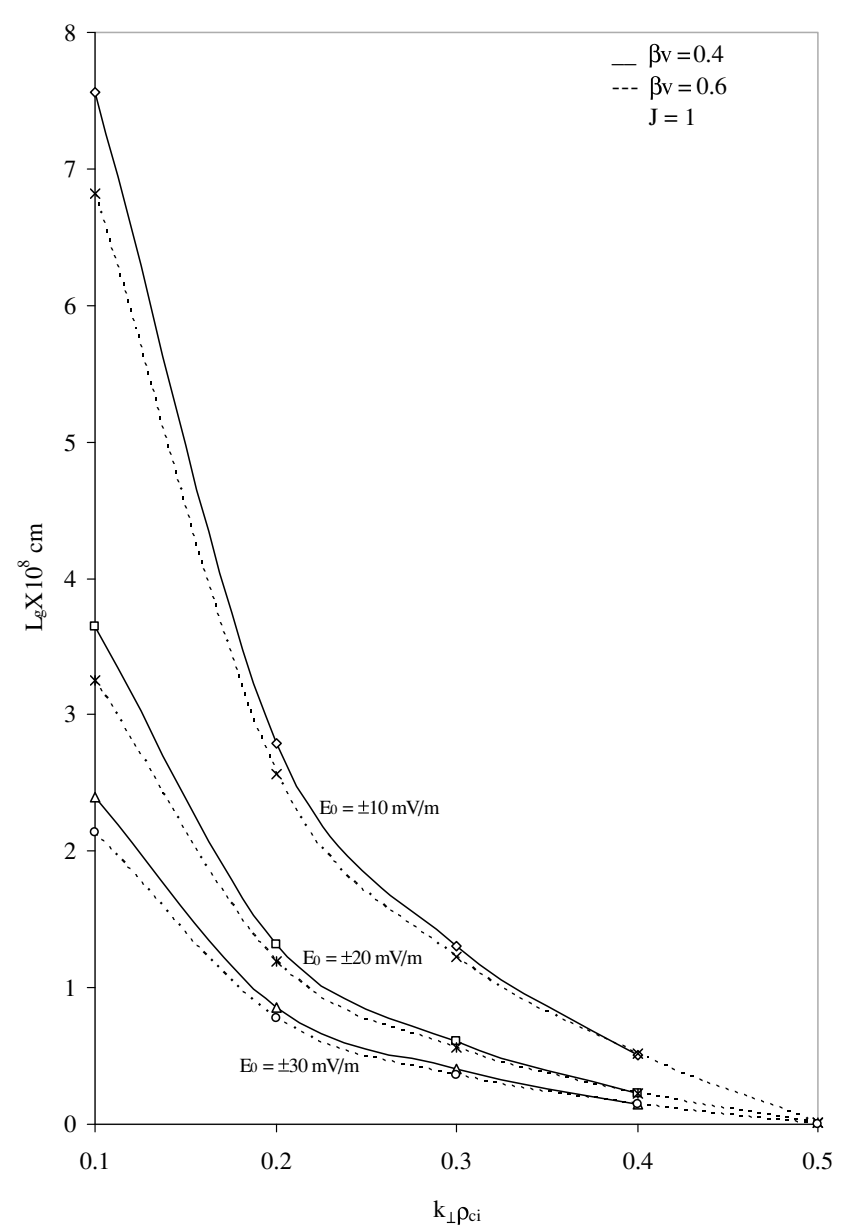

Fig. 10. Variation in growth length $\left(L_{g}\right)$ with perpendicular wave number $\left(k_{\perp} \rho_{c i}\right)$ for different values of parallel electric field $\left(E_{0}\right)$ and strong shear $(\beta v)$ with $J=1$.

bution index $(J)$, parallel electric field $\left(E_{0}\right)$ and thermal anisotropy $(\varepsilon)$ on the frequency and growth length of the $\mathrm{KAW}$ in the strong shear regime $(\beta v=0.4$ and 0.6$)$.

Figure 7 shows the variation in wave frequency $\left(\frac{\omega}{\omega_{c i}}\right)$ with the perpendicular wave number $\left(k_{\perp} \rho_{c i}\right)$ for different values of the loss-cone distribution index $(J)$ in the strong shear regime $(\beta v)$. The loss-cone distribution index $(J)$ decreases the frequency of the KAW, as in case of weak shear. The increasing values of strong shear enhance its frequency. The negative values of the frequency are due to the parallel electric field $\left(E_{0}\right)$ in the strong shear regime.

Figure 8 shows the variation in the growth length $\left(L_{g}\right)$ with perpendicular wave number $\left(k_{\perp} \rho_{c i}\right)$ for different values of the loss-cone distribution index $(J)$ in the strong shear regime. It is observed that in the strong shear regime the loss-cone distribution index $(J)$ reduces the growth length of the wave, possibly in relation with the decreasing width of the loss-cone. Hence, an increased number of particles may be available to provide energy to the wave. Increasing values of shear also decrease the growth length of the wave.

Figure 9 shows the variation in the frequency of the wave $\left(\frac{\omega}{\omega_{c i}}\right)$ with the perpendicular wave number $\left(k_{\perp} \rho_{c i}\right)$ for the different values of the parallel electric field $\left(E_{0}\right)$ and strong

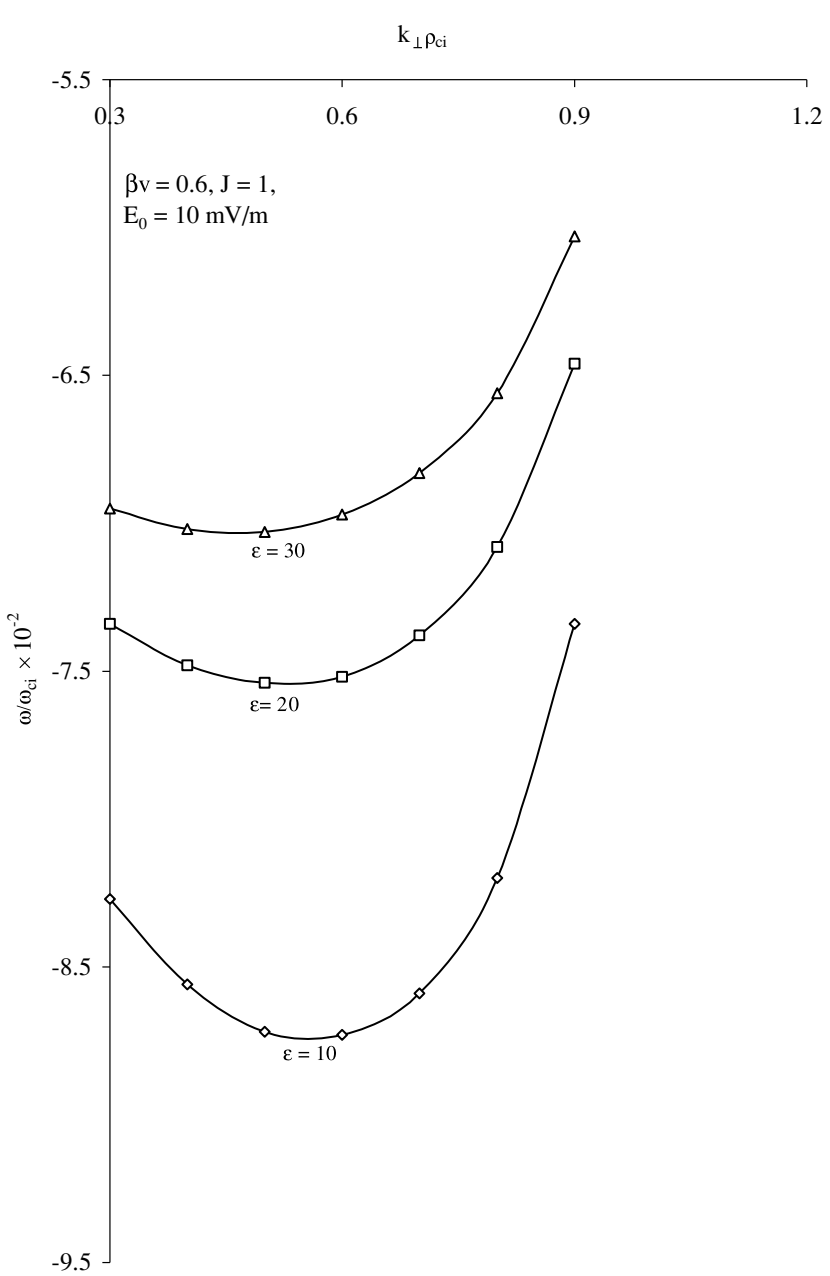

Fig. 11. Variation in wave frequency $\left(\frac{\omega}{\omega_{c i}}\right)$ with perpendicular wave number $\left(k_{\perp} \rho_{c i}\right)$ for different values of ion thermal anisotropy $(\varepsilon)$ with $J=1, \beta v=0.6$ and $E_{0}=10 \mathrm{mV} / \mathrm{m}$.

shear $(\beta v)$. It is observed that the earthward (positive) parallel electric field enhances the frequency of the reflected wave, whereas the tailward (negative) parallel electric field enhances the frequency of the incident wave. The increasing values of the strong shear further enhance this effect. The opposite effects of parallel electric field are observed, as compared to the case of weak shear (Fig. 3).

Figure 10 shows the variation in growth length $\left(L_{g}\right)$ with perpendicular wave number $\left(k_{\perp} \rho_{c i}\right)$ for different values of the parallel electric field $\left(E_{0}\right)$ in the strong shear regime $(\beta v)$ with $J=1$. It is observed that the effect of increasing values of the parallel electric field $\left(E_{0}\right)$ is to lower the growth length of the wave, which means that the wave attains sufficient amplitude quickly to propagate towards the auroral ionosphere in the presence of the parallel electric field. Figure 10 also shows that an increasing value of the strong shear $(\beta v)$ contributes towards this effect.

Thus, during the expansion phase of the substorm, when the shear is strong, the parallel electric field may lower the growth length $\left(L_{g}\right)$ of KAW.

Figures 11 and 12 show the variation in wave frequency $\left(\frac{\omega}{\omega_{c i}}\right)$ and growth length $\left(L_{g}\right)$ with the perpendicular wave number $\left(k_{\perp} \rho_{c i}\right)$ for different values of the ther- 


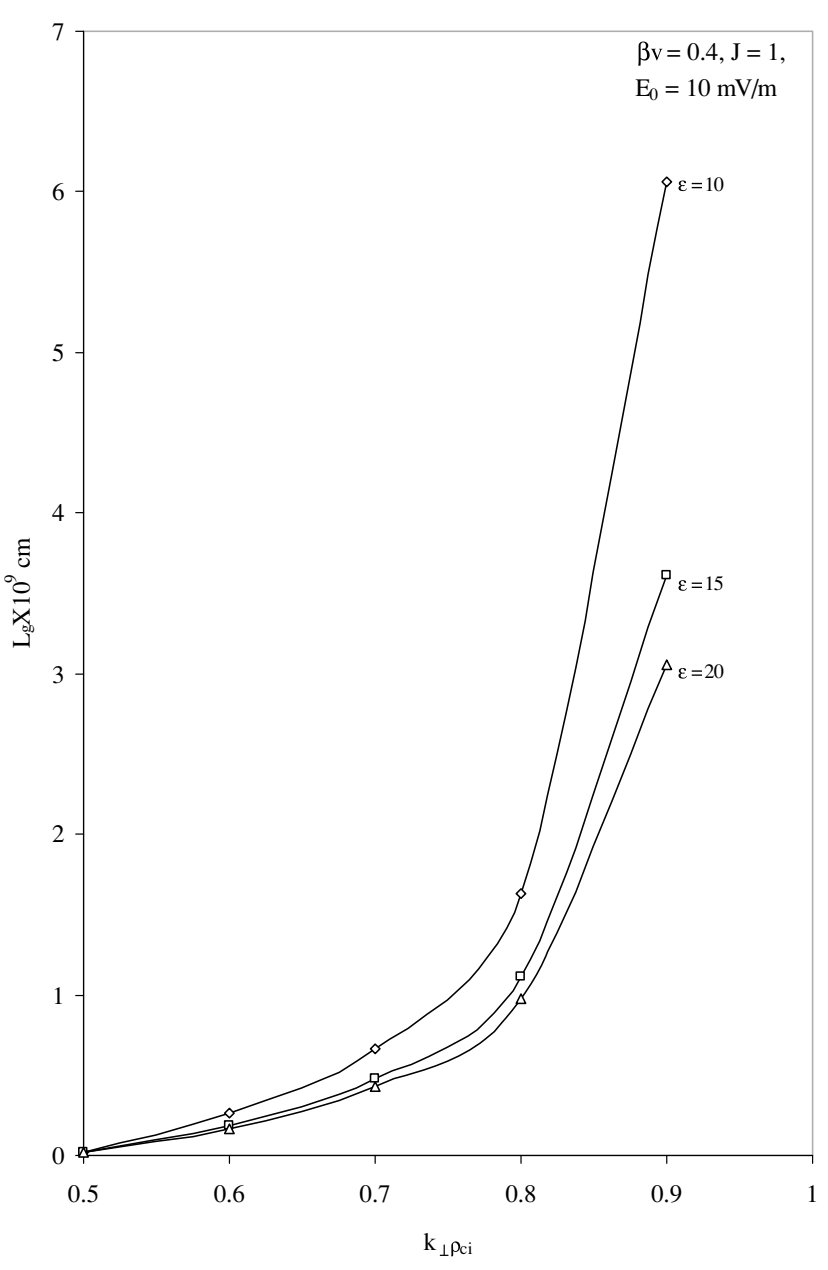

Fig. 12. Variation in growth length $\left(L_{g}\right)$ with perpendicular wave number $\left(k_{\perp} \rho_{c i}\right)$ for different values of ion thermal anisotropy $(\varepsilon)$ with $J=1$, $\beta v=0.4$ and $E_{0}=10 \mathrm{mV} / \mathrm{m}$.

mal anisotropy $(\varepsilon)$ in the strong shear regime. It is observed that the thermal anisotropy lowers the frequency and growth length of the KAW at small obliqueness angles. Thus, the thermal anisotropy may lower the frequency band of the KAW.

The results of the present study are consistent with the findings of Wu and Seyler (2003). Wu and Seyler (2003) have shown that the Alfven wave can be generated and stabilised by the sheared flow. They have also predicted that when the Richardson number $R_{i}=\frac{k_{z}^{2}}{k_{y}^{2} V_{0}^{12}}$ (paragraph 47, Wu and Seyler, 2003) is $0<R_{i}<1 / 4$, the wave grows, whereas when $R_{i}>1 / 4$, the wave is Landau-damped. The results of the present study show that the KAW may be generated at the time of strong shear and may be stabilised at the time of weak shear due to cyclotron interaction of the KAW. Analytical results obtained here also show this. Equations (25) and (27) show that in both the weak and strong shear regimes, the resonant electron associated with the term $i \sqrt{\frac{\pi}{2}} \frac{\omega}{k_{z} v_{t e z}}$ act as a source of free energy for the KAW. However, the ion longitudinal motion associated with the term $v_{t i z}^{2}$ suppresses the instability in the weak shear regime.

The results of the present investigation may also be applicable to laboratory plasma where the flow is mostly inho- mogeneous and therefore encounters velocity shear in varying magnitudes and to mirror-like devices, which have the loss-cone distribution of particles.

\section{Conclusions}

The following conclusions can be drawn from the results of the present study:

1. Energetic KAWs with enhanced frequency may be generated by the shear in the PSBL at the substorm onset due to the cyclotron interaction.

2. The loss-cone distribution index decreases the frequency band but enhances the growth length $\left(L_{g}\right)$ of the KAW in the weak shear regime, whereas in the strong shear regime, the loss-cone distribution index lowers the growth length of the KAW.

3. The electric field along the magnetic lines enhances the frequency of the KAW but decreases the growth length of the KAW in the case of weak shear and strong shear.

4. The parallel electric field may give rise to the reflected KAWs, which may then superimpose with the incident KAW to give the observed large-amplitude travelling waves and/or standing waves at the substorm times.

5. The parallel electric field associated with the energetic KAWs in addition to the external parallel electric field at the substorm onset may heat the field-aligned electrons interacting with the wave, leading to the parallel electron energisation that ultimately causes intense aurora.

Acknowledgments. The financial assistance of the Council of Scientific and Industrial Research (CSIR), India to Ruchi Mishra and Department of Science and Technology (DST), India to P. Varma is thankfully acknowledged.

\section{References}

Ahirwar, G., P. Varma, and M. S. Tiwari, Electromagnetic ion-cyclotron instability in the presence of a parallel electric field with general distribution function-Particle aspect analysis, Ann. Geophys., 24, 1919, 2006.

Angelopoulos, V., J. A. Chapman, F. S. Mozer, J. D. Sudder, C. T. Russell, K. Tsuruda, T. Mukai, T. J. Hughes, and K. Y. Yumoto, Plasma sheet electromagnetic power generation and its dissipation along auroral field lines, J. Geophys. Res., 107, doi: 10.1029/2001 JA900136, SMP 14, 120, 2002.

Duan, S. P., Z. Y. Li, and Z. X. Liu, Kinetic Alfven wave driven by the density inhomogeneity in the presence of loss-cone distribution function-particle aspect analysis, Planet. Space Sci., 53, 1167, 2005.

Eastman, T. E., L. A. Frank, W. K. Peterson, and W. Lennartsson, The plasma sheet boundary layer, J. Geophys. Res., 89, 1553, 1984.

Ergun, R. E., Y. J. Su, L. Andersson, C. W. Carlson, J. P. Mc Fadden, F. S. Mozer, D. L. Newmann, M. V. Goldman, and R. J. Strangeway, Direct observations of localized parallel electric fields in a space plasma, Phys. Rev. Lett., 87, 5003, 2001.

Ganguli, G., S. Slinker, V. Gavrishchaka, and W. Scales, Low frequency oscillations in plasma with spatially variable field-aligned flow, Phys. Plasmas, 9, 2321, 2002.

Gavrishchaka, V., M. E. Koepke, and G. Ganguli, Dispersive properties of a magnetised plasma with a field aligned drift and inhomogeneous transverse flow, Phys. Plasmas, 3, 3091, 1996.

Gavrishchaka, V. V., G. Ganguli, W. Scales, S. P. Slinker, C. C. Chatson, J. P. McFadden, R. E. Ergun, and C. W. Carlson, Multiscale coherent structures and broadband waves due to parallel inhomogeneous flows, Phys. Rev. Lett., 85, 4285, 2000.

Gomberoff, L. and S. Cuperman, On the kinetic instability of uniform plasmas with generalized loss-cone distribution function, J. Plasma Phys., 25, 99, 1981. 
Hamrin, M., M. Andre, G. Ganguli, V. Gavrishchaka, E. Koepke, M. W. Zintl, N. Ivchenko, T. Karlsson, and J. H. Clemmons, Inhomogeneous transverse electric fields and wave generation in the auroral region: A statistical study, J. Geophys. Res., 106, 10803, 2001.

Hesegawa, A. and L. Chen, Kinetic process of plasma heating due to Alfven wave excitation, Phys. Res. Lett., 35, 370, 1975.

Hull, A. J., J. W. Bonnell, F. S. Mozer, J. D. Scudder, and C. C. Chaston, Large parallel electric fields in the upward current region of the aurora: Evidence for ambipolar effects, J. Geophys. Res., 108(A6), 1265, 2003.

Keiling, A., J. R. Wygant, C. Cattell, M. Temerin, F. S. Mozer, C. A. Kletzing, J. Sudder, C. T. Russell, W. Lotko, and A. V. Streltsov, Large Alfven wave power in the plasma sheet boundary layer during the expansion phase of substorm, Geophys. Res. Lett., 27, 3169, 2000.

Keiling, A., J. R. Wygant, C. A. Cattell, W. Peria, G. Parks, M. Temerin, F. S. Mozer, C. T. Russell, and C. A. Kletzing, Correlation of Alfven wave Poynting flux in the plasma sheet at 4-7 $R_{E}$ with ionospheric electron energy flux, J. Geophys. Res., 107(A7), 1132, 2002.

Keiling, A., G. K. Parks, J. R. Wygant, J. Dombeck, F. S. Mozer, C. T. Russell, A. V. Streltsov, and W. Lotko, Some properties of Alfven waves: Observations in the tail lobes and the plasma sheet boundary layer, J. Geophys. Res., 110, A10S11, 1-16, 2005.

Lysak, R. L. and W. Lotko, On the kinetic dispersion relation for shear Alfven waves, J. Geophys. Res., 101, 5085, 1996.

Mishra, R. and M. S. Tiwari, Effect of parallel electric field on electrostatic ion-cyclotron instability in anisotropic plasma in the presence of ion beam and general distribution function-Particle aspect analysis, Planet. Space Sci., 54, 188-199, 2006.

Nakamura, R., W. Banmjohann, T. Nagai, M. Fujimoto, T. Mukai, B. Klecker, R. Treumann, A. Balogh, H. Reme, J. A. Sanvand, L. Kistler, C. Monikis, C. J. Owen, A. N. Fazakerley, J. P. Dewhurst, and Y. Bogdanova, Flow shear near the boundary of the plasma sheet observed by Cluster and Geotail, J. Geophys. Res., 109, A05204/1-12, doi: 10.1029/2003 JA010174, 2004.
Pines, D. and R. Schrieffer, Collective behaviour in solid-state, Plasmas Phys. Rev., 124, 1387, 1961.

Scime, E. E., R. Murphy, G. I. Ganguli, and E. Edlund, Electrostatic ioncyclotron waves in a currentless, anisotropic plasma with inhomogeneous flow, Phys. Plasmas, 10, 4609, 2003.

Summers, D. and R. M. Thorne, Plasma microinstability driven by losscone distribution, J. Plasma Phys., 53, 293, 1995.

Tiwari, B. V., R. Mishra, P. Varma, and M. S. Tiwari, Generation of kinetic Alfven wave by velocity shear in the plasma sheet boundary layer during substorm, Indian J. Pure Appl. Phys., 44, 917, 2006.

Vogt, J. and G. Haerendel, Reflection and transmission of Alfven waves at the auroral acceleration region, Geophys. Res. Lett., 25, 277, 1998.

Wang, X. Y., Z. X. Liu, Z. Y. Li, and X. B. Zhang, Kinetic Alfven waves driven by velocity shear, Phys. Plasmas, 5, 836, 1998.

$\mathrm{Wu}, \mathrm{K}$. and C. E. Seyler, Instability of inertial Alfven waves in transverse sheared flow, J. Geophys. Res., 108(A6), 1236, SMP 9, 1-19, 2003.

Wygant, J. R., A. Keiling, C. A. Cattell, M. Johnson, R. L. Lysak, M. Temerin, F. S. Mozar, C. A. Kletzing, J. D. Scudder, W. Peterson, C. T. Russell, G. Parks, M. Brittnacher, G. Germany, and J. Spann, Polar spacecraft based comparisons of intense electric fields and Poynting flux near and within the plasma sheet tail lobe boundary to UVI images: An energy source for the aurora, J. Geophys. Res., 105, 18675, 2000.

Wygant, J. R., A. Keiling, C. A. Cattell, R. L. Lysak, M. Temerin, F. S. Mozer, C. A. Kletzing, J. D. Scudder, V. Streltsov, W. Lotko, and C. T. Russell, Evidence for kinetic Alfven waves and parallel electron energisation at 4-6 $R_{E}$ altitudes in the plasma sheet boundary layer, $J$. Geophys. Res., 107(A8), SMP 24-1, 2002.

B. V. Tiwari, R. Mishra, P. Varma (e-mail: poornimavarma@yahoo. com), and M. S. Tiwari (e-mail: tiwarims@rediffmail.com) 